\title{
The odd Weibull-Topp-Leone-G power series family of dis- tributions: model, properties, and applications
}

\author{
Broderick Oluyede $^{\mathrm{a}}$, Fastel Chipepa ${ }^{\mathrm{a}, \mathrm{b}, *}$, Divine Wanduku ${ }^{\mathrm{c}}$ \\ ${ }^{a}$ Department of Mathematical Statistics, Botswana International University of Science and Technology, P. Bag 16, Palapye, Botswana. \\ ${ }^{b}$ Department of Applied Mathematics and Statistics, Midlands State University, P. Bag 9055, Gweru, Zimbabwe. \\ ${ }^{c}$ Department of Mathematical Sciences, Georgia Southern University, GA 30460, USA.
}

\begin{abstract}
A new generalization of the odd Weibull-Topp-Leone-G family of distributions called the odd Weibull-Topp-Leone-G power series family of distributions is developed. Statistical properties of the new distribution were derived. We also derive the maximum likelihood estimates of the proposed model. Some special cases for the new family of distributions were also considered. We conducted a simulation study to evaluate the consistency of the maximum likelihood estimates. Two real data examples were also considered to demonstrate the usefulness of the newly proposed family of distributions.
\end{abstract}

Keywords: Odd Weibull-Topp-Leone-G, odd Weibull-G, Topp-Leone-G distribution, power series distribution. 2020 MSC: 62E99, 60E05.

(C)2021 All rights reserved.

\section{Introduction}

Power series distributions are widely used in finance and actuarial science. Power series generalizations include the double bounded Kumaraswamy-power series class of distributions by [7], complementary extended Weibull power series class of distributions by [15], compound class of Weibull and power series distributions by [26], generalized exponential power series distributions by [24], compound class of linear failure rate power series distributions by [25], Burr XII power series distributions by [31], generalized linear failure rate power series distribution by [19], compound class of extended Weibull power series distributions by [30].

Some generalizations of the Topp-Leone distribution includes the Topp-Leone-G family by [5], ToppLeone generated Weibull distribution by [6], transmuted Topp-Leone Weibull lifetime distribution [20], Topp-Leone generalized inverted exponential distribution [4] and Topp-Leone-Marshall-Olkin-G by [11]. Odd generalized families includes the odd Lindley-G distribution by [16], odd generalized half logistic Weibull-G by [10], odd log-logistic Lindley-G by [3], odd exponentiated half logistic Burr XII distribution by [2], to mention a few.

\footnotetext{
*Corresponding author

Email addresses: oluyedeo@biust.ac.bw (Broderick Oluyede), chipepaf@staff.msu.ac.zw (Fastel Chipepa), dwanduku@georgiasouthern.edu (Divine Wanduku)
}

doi: $10.22436 /$ jnsa.014.04.06

Received: 2020-10-22 Revised: 2020-11-15 Accepted: 2020-12-02 
Recently, [8] developed a new family of distributions called the odd Weibull-Topp-Leone-G (OW-TLG) family of distributions using the generalized Weibull family by [18] and the Topp-Leone-G distribution. The generalized Weibull family distribution have cumulative distribution function (cdf) and probability density function (pdf) given by

$$
\mathrm{G}(x ; \xi)=1-\exp [-\alpha \mathrm{H}(x, \xi)]
$$

and

$$
g(x ; \xi)=\alpha \exp [-\alpha H(x ; \xi)] h(x ; \xi),
$$

respectively for a parameter vector $\xi$. The Topp-Leone-G (TL-G) family of distributions by [5] have cdf given by

$$
\mathrm{G}_{\mathrm{TL}-\mathrm{G}}(x, \gamma, \xi)=\left[1-\overline{\mathrm{G}}^{2}(x ; \xi)\right]^{\gamma},
$$

for $\gamma>0$ and parameter vector $\xi$. In the OW-TL-G family of distributions $\alpha \mathrm{H}(x ; \xi)$ in equation (1.1) was replaced by $\left[\frac{\left[1-\bar{G}^{2}(x ; \xi)\right]^{\gamma}}{\left[1-\left(1-\bar{G}^{2}(x ; \xi)\right)^{\gamma}\right]}\right]^{\alpha}$ (the exponentiated odds of the TL-G distribution), so that the survival function of the OW-TL-G family of distributions is given by

$$
\overline{\mathrm{F}}(\mathrm{x} ; \alpha, \gamma, \xi)=\exp \{-\mathrm{t}\},
$$

where $t=\left[\frac{\left[1-\bar{G}^{2}(x ; \xi)\right]^{\gamma}}{\left[1-\left(1-\bar{G}^{2}(x ; \xi)\right)^{\gamma}\right]}\right]^{\alpha}$, for $\alpha, \gamma>0$ and parameter vector $\xi$.

In this note, we are motivated by the desirable properties exhibited by the power series generalizations in terms of data fitting, to develop a new odd Weibull-Topp-Leone-G power series family of distributions. The new proposed distribution exhibit flexibility in data fitting as the hazard rate function takes both monotonic and non-monotonic shapes. We hope the new distribution will receive attention from other researchers in the field of science and engineering. The distribution fit lifetime and reliability data well as demonstrated in this paper.

Let $\mathrm{N}$ be a discrete random variable following a power series distribution assumed to be truncated at zero, whose probability mass function (pmf) is given by

$$
P(N=n)=\frac{a_{n} \theta^{n}}{C(\theta)}, \quad n=1,2, \ldots,
$$

where $C(\theta)=\sum_{n=1}^{\infty} a_{n} \theta^{n}$ is finite, $\theta>0$, and $\left\{a_{n}\right\}_{n} \geqslant 1$ a sequence of positive real numbers. The power series family of distributions includes binomial, Poisson, geometric and logarithmic distributions [21]. Table 1 shows some useful quantities including $a_{n}, C(\theta), C^{-1}(\theta), C^{\prime}(\theta)$ and $C^{\prime \prime}(\theta)$ for the binomial, Poisson, geometric, and logarithmic distributions.

Table 1: Useful quantities for some power series distributions.

\begin{tabular}{lcccccc}
\hline Distribution & $\mathrm{C}(\theta)$ & $\mathrm{C}^{\prime}(\theta)$ & \multicolumn{1}{c}{$\mathrm{C}^{\prime \prime}(\theta)$} & $\mathrm{C}^{-1}(\theta)$ & $\mathrm{a}_{\mathrm{n}}$ & Parameter Space \\
\hline Binomial & $(1+\theta)^{\mathrm{m}}-1$ & $\mathrm{~m}(1+\theta)^{\mathrm{m}-1}$ & $\mathrm{~m}(\mathrm{~m}-1)(1+\theta)^{\mathrm{m}-2}$ & $(\theta-1)^{1 / m}-1$ & $\left(\begin{array}{l}\mathrm{m} \\
n\end{array}\right)$ & $(0,1)$ \\
Poisson & $e^{\theta}-1$ & $e^{\theta}$ & $e^{\theta}$ & $\log (1+\theta)$ & $(\mathrm{n} !)^{-1}$ & $(0, \infty)$ \\
Geometric & $\theta(1-\theta)^{-1}$ & $(1-\theta)^{-2}$ & $2(1-\theta)^{-3}$ & $\theta(1+\theta)^{-1}$ & 1 & $(0,1)$ \\
Logarithmic & $-\log (1-\theta)$ & $(1-\theta)^{-1}$ & $(1-\theta)^{-2}$ & $1-e^{-\theta}$ & $n^{-1}$ & $(0,1)$ \\
\hline
\end{tabular}

Let $X=Y_{(1)}=\min \left(Y_{1}, \ldots, Y_{N}\right)$. The conditional distribution of $X$ given $N=n$ is given by

$$
\mathrm{G}_{X \mid N=n}(x)=1-\prod_{i=1}^{n}(1-G(x))=1-S^{n}(x),
$$

where $G(x)=G(x ; \alpha, \gamma, \xi)$ is the OW-TL-G cdf. The cdf of the life length of the whole system, $X, F_{\theta}$, is given by

$$
F_{\theta}(x)=1-\frac{C(\theta S(x))}{C(\theta)}
$$


where $S(x)$ is the survival function of the OW-TL-G family of distributions. The corresponding probability density function (pdf) is given by

$$
f_{\theta}(x)=\frac{d F_{\theta}(x)}{d x}=\frac{\theta g(x) C^{\prime}(\theta S(x))}{C(\theta)}
$$

The hazard and reverse hazard rate functions are given by

$$
h_{\theta}(x)=\frac{f_{\theta}(x)}{S_{\theta}(x)}=\theta g(x) \frac{C^{\prime}(\theta S(x))}{C(\theta S(x))}, \quad \text { and } \quad \tau_{\theta}(x)=\frac{f_{\theta}(x)}{F_{\theta}(x)}=\theta g(x) \frac{C^{\prime}(\theta S(x))}{C(\theta)-C(\theta S(x))^{\prime}},
$$

respectively.

The rest of the paper is organized as follows. We develop the new model and statistical properties in Section 2. We do maximum likelihood estimation in Section 3. Some special families are presented in Section 4. A simulation study is conducted in Section 5. We present two real data examples in Section 6 and concluding remarks in Section 7.

\section{The model and properties}

A new power series family of distributions, namely, the odd Weibull-Topp-Leone-G power series (OW-TL-GPS) family of distributions is developed in this section. Some statistical properties for the proposed distribution are also presented.

\subsection{The model}

We derive the new family of distributions from the generalizations given in equations (1.2), (1.3), and (1.4). Therefore, the cdf and pdf of the OW-TL-GPS distribution are given by

$$
F_{\theta}(x)=\sum_{n=1}^{\infty} \frac{a_{n} \theta^{n}}{C(\theta)}(1-\exp \{-t\})=1-\frac{C(\theta(\exp \{-t\}))}{C(\theta)}
$$

and

$$
f_{\theta}(x)=\frac{2 \theta \gamma \alpha g(x ; \xi) \bar{G}(x ; \xi)\left[1-\bar{G}^{2}(x ; \xi)\right]^{\gamma \alpha-1}}{\left[1-\left(1-\bar{G}^{2}(x ; \xi)\right)^{\gamma}\right]^{\alpha+1}} \exp \{-t\} \frac{C^{\prime}(\theta(\exp \{-t\}))}{C(\theta)},
$$

respectively, for $\alpha, \gamma, \theta>0, G(x ; \xi)$ is the baseline distribution with parameter vector $\xi$ and $\bar{G}(x ; \xi)=1-G(x ; \xi)$. Other sub-families of the OW-TL-GPS family of distributions are obtained by changing the baseline distribution function $\mathrm{G}(\mathrm{x} ; \xi)$ and the power series distribution.

Table 2 shows some special cases of the OW-TL-GPS distribution. The hazard and reverse hazard rate functions

Table 2: Special cases of the OW-TL-GPS.

\begin{tabular}{llll}
\hline Distribution & $\mathrm{a}_{\mathrm{n}}$ & $\mathrm{C}(\theta)$ & $\mathrm{cdf}$ \\
\hline OW-TL-G Poisson & $(\mathrm{n} !)^{-1}$ & $\mathrm{e}^{\theta}-1$ & $1-\frac{\exp \{-\mathrm{t}\}-1}{e^{\theta}-1}$ \\
OW-TL-G Geometric & 1 & $\theta(1-\theta)^{-1}$ & $1-\frac{(1-\theta) \exp \{-\mathrm{t}\}}{1-\theta \exp \{-\mathrm{t}\}}$ \\
OW-TL-G Logarithmic & $\mathrm{n}^{-1}$ & $-\log (1-\theta)$ & $1-\frac{\log (1-\theta(\exp \{-\mathrm{t}\}))}{\log (1-\theta)}$ \\
OW-TL-G Binomial & $\left(\begin{array}{c}\mathrm{m} \\
\mathrm{n}\end{array}\right)$ & $(1+\theta)^{\mathrm{m}}-1$ & $1-\frac{(1+\theta \exp \{-\mathrm{t}\}))^{\mathrm{m}}-1}{(1+\theta)^{\mathrm{m}-1}}$ \\
\hline
\end{tabular}

of the OW-TL-GPS family of distributions are given by

$$
h_{\theta}(x)=\frac{2 \gamma \theta \beta g(x ; \xi) \bar{G}(x ; \xi)\left[1-\bar{G}^{2}(x ; \xi)\right]^{\gamma \alpha-1}}{\left[1-\left(1-\bar{G}^{2}(x ; \xi)\right)^{\gamma}\right]^{\alpha+1}} \exp \{-t\} \frac{C^{\prime}(\theta \exp \{-t\})}{C(\theta \exp \{-t\})}
$$

and

$$
\tau_{\theta}(x)=\frac{2 \gamma \theta \alpha g(x ; \xi) \bar{G}(x ; \xi)\left[1-\bar{G}^{2}(x ; \xi)\right]^{\gamma \alpha-1}}{\left[1-\left(1-\bar{G}^{2}(x ; \xi)\right)^{\gamma}\right]^{\alpha+1}} \exp \{-t\} \frac{C^{\prime}(\theta \exp \{-t\})}{C(\theta)-C(\theta \exp \{-t\})},
$$

respectively. 


\subsection{Expansion of the density function}

In this section, we derive the linear representation of the OW-TL-GPS family of distributions. Equation (2.1) can be written as

$$
f_{\theta}(x)=\sum_{n=1}^{\infty} \frac{n a_{n} \theta^{n}}{C(\theta)} \frac{2 \gamma \alpha g(x ; \xi) \bar{G}(x ; \xi)\left[1-\bar{G}^{2}(x ; \xi)\right]^{\gamma \alpha-1}}{\left[1-\left(1-\bar{G}^{2}(x ; \xi)\right)^{\gamma}\right]^{\alpha+1}} \exp \left\{-n\left[\frac{\left[1-\bar{G}^{2}(x ; \xi)\right]^{\gamma}}{\left[1-\left(1-\bar{G}^{2}(x ; \xi)\right)^{\gamma}\right]}\right]^{\alpha}\right\} .
$$

Considering the following expansions

$$
\begin{aligned}
& \exp \left\{-n\left[\frac{\left[1-\bar{G}^{2}(x ; \xi)\right]^{\gamma}}{\left[1-\left(1-\bar{G}^{2}(x ; \xi)\right)^{\gamma}\right]}\right]^{\alpha}\right\}=\sum_{l=0}^{\infty} \frac{(-1)^{l} n^{l}}{l !}\left[\frac{\left[1-\bar{G}^{2}(x ; \xi)\right]^{\gamma \alpha l}}{\left[1-\left(1-\bar{G}^{2}(x ; \xi)\right)^{\gamma}\right]^{\alpha l}}\right], \\
& {\left[1-\left(1-\overline{\mathrm{G}}^{2}(\mathrm{x} ; \xi)\right)^{\gamma}\right]^{-(\alpha(\mathrm{l}+1)+1)}=\sum_{w=0}^{\infty}(-1)^{w}\left(\begin{array}{c}
-(\alpha(\mathrm{l}+1)+1) \\
w
\end{array}\right)\left(1-\overline{\mathrm{G}}^{2}(\mathrm{x} ; \xi)\right)^{\gamma w},} \\
& \left(1-\overline{\mathrm{G}}^{2}(x ; \xi)\right)^{\alpha \gamma(l+1)+\gamma w-1}=\sum_{\mathrm{h}=0}^{\infty}(-1)^{\mathrm{h}}\left(\begin{array}{c}
\alpha \gamma(l+1)+\gamma w-1 \\
\mathrm{~h}
\end{array}\right) \overline{\mathrm{G}}(x ; \xi)^{2 h},
\end{aligned}
$$

and

$$
\overline{\mathrm{G}}(x ; \xi)^{2 \mathrm{~h}+1}=\sum_{\mathrm{m}=0}^{\infty}(-1)^{\mathrm{m}}\left(\begin{array}{c}
2 \mathrm{~h}+1 \\
\mathrm{~m}
\end{array}\right) \mathrm{G}^{\mathrm{m}}(x ; \xi)
$$

yields

$$
\begin{aligned}
f_{\theta}(x)= & \sum_{l, w, h, m=0}^{\infty} \sum_{n=1}^{\infty} \frac{(-1)^{l+w+h+m_{n}{ }^{l+1} 2 \gamma \alpha a_{n} \theta^{n}}}{l ! C(\theta)}\left(\begin{array}{c}
-(\alpha(l+1)+1) \\
w
\end{array}\right)\left(\begin{array}{c}
\alpha \gamma(l+1)+\gamma w-1 \\
h
\end{array}\right) \\
& \times\left(\begin{array}{c}
2 h+1 \\
m
\end{array}\right) g(x ; \xi) G^{m}(x ; \xi)=\sum_{m=0}^{\infty} \phi_{m} g_{m}(x ; \xi),
\end{aligned}
$$

where

$$
\phi_{m}=\sum_{l, w, h=0}^{\infty} \sum_{n=1}^{\infty} \frac{(-1)^{l+w+h+m} n^{l+1} 2 \gamma \alpha a_{n} \theta^{n}}{l !(m+1) C(\theta)}\left(\begin{array}{c}
-(\alpha(l+1)+1) \\
w
\end{array}\right)\left(\begin{array}{c}
\alpha \gamma(l+1)+\gamma w-1 \\
h
\end{array}\right)\left(\begin{array}{c}
2 h+1 \\
m
\end{array}\right)
$$

and $g_{m}(x ; \xi)=(m+1) g(x ; \xi) G^{m}(x ; \xi)$ is an exponentiated-G (Exp-G) density with power parameter $m$. Thus, the OW-TL-GPS density can be expressed as a linear combination of Exp-G densities. Therefore, we can derive other statistical properties of the OW-TL-GPS distribution directly from the statistical properties of the Exp-G distribution.

\subsection{Quantile function}

The quantile function of the OL-GPS distribution is obtained by inverting $F_{\theta}(x)=u, 0 \leqslant u \leqslant 1$. Note that

$$
u=1-\frac{C\left(\theta\left(\exp \left\{-\left[\frac{\left[1-\overline{\mathrm{G}}^{2}(x ; \xi)\right]^{\gamma}}{\left[1-\left(1-\overline{\mathrm{G}}^{2}(x ; \xi)\right)^{\gamma}\right]}\right]^{\alpha}\right\}\right)\right)}{C(\theta)}
$$

simplifies to

$$
\ln \left(\frac{\mathrm{C}^{-1}(\mathrm{C}(\theta)(1-\mathrm{u}))}{\theta}\right)=-\left[\frac{\left[1-\overline{\mathrm{G}}^{2}(\mathrm{x} ; \xi)\right]^{\gamma}}{\left[1-\left(1-\overline{\mathrm{G}}^{2}(\mathrm{x} ; \xi)\right)^{\gamma}\right]}\right]^{\alpha},
$$

which further simplifies to

$$
\bar{G}^{2}(x ; \xi)=1-\left[\frac{\left(-\ln \left[\frac{\mathrm{C}^{-1}(\mathrm{C}(\theta)(1-\mathfrak{u}))}{\theta}\right]\right)^{1 / \alpha}}{1+\left(-\ln \left[\frac{\mathrm{C}^{-1}(\mathrm{C}(\theta)(1-\mathfrak{u}))}{\theta}\right]\right)^{1 / \alpha}}\right]^{1 / \gamma},
$$


such that

$$
\mathrm{G}(x ; \xi)=1-\left(1-\left[\frac{\left(-\ln \left[\frac{\mathrm{C}^{-1}(\mathrm{C}(\theta)(1-\mathfrak{u}))}{\theta}\right]\right)^{1 / \alpha}}{1+\left(-\ln \left[\frac{\mathrm{C}^{-1}(\mathrm{C}(\theta)(1-\mathfrak{u}))}{\theta}\right]\right)^{1 / \alpha}}\right]^{1 / \gamma}\right)^{1 / 2} .
$$

Hence, we obtain the quantile values of the OW-TL-GPS family of distributions by solving the non-linear equation

$$
\mathrm{Q}_{\mathrm{F}}(\mathrm{u})=\mathrm{G}^{-1}\left[1-\left(1-\left[\frac{\left(-\ln \left[\frac{\mathrm{C}^{-1}(\mathrm{C}(\theta)(1-\mathfrak{u}))}{\theta}\right]\right)^{1 / \alpha}}{1+\left(-\ln \left[\frac{\mathrm{C}^{-1}(\mathrm{C}(\theta)(1-\mathfrak{u}))}{\theta}\right]\right)^{1 / \alpha}}\right]^{1 / \gamma}\right)^{1 / 2}\right]
$$

using numerical methods with the aid of statistical software such as R, SAS, and MATLAB. Table 3 shows the quantile values for the odd Weibull-Topp-Leone-log logistic Poisson (OW-TL-LLoGP) distribution.

Table 3: Table of quantiles for selected parameters of OW-TL-LLoGP distribution.

\begin{tabular}{l|ccccc}
\hline $\mathrm{u}$ & $(0.5,1.5,0.5,1.5)$ & $(0.5,1,0.5,1.2)$ & $(1.1,2.5,0.5,2.5)$ & $(0.5,1.5,0.5,0.5)$ & $(1.1,0.9,1,1.1)$ \\
\hline 0.1 & 0.0557 & 0.0079 & 0.5244 & 0.0002 & 0.0450 \\
0.2 & 0.0974 & 0.0246 & 0.5828 & 0.0009 & 0.0907 \\
0.3 & 0.1353 & 0.0480 & 0.6224 & 0.0025 & 0.1410 \\
0.4 & 0.1722 & 0.0779 & 0.6545 & 0.0051 & 0.1980 \\
0.5 & 0.2096 & 0.1147 & 0.6830 & 0.0092 & 0.2642 \\
0.6 & 0.2493 & 0.1604 & 0.7102 & 0.0155 & 0.3441 \\
0.7 & 0.2935 & 0.2185 & 0.7378 & 0.0253 & 0.4451 \\
0.8 & 0.3467 & 0.2972 & 0.7683 & 0.0417 & 0.5837 \\
0.9 & 0.4214 & 0.4217 & 0.8073 & 0.0748 & 0.8090 \\
\hline
\end{tabular}

\subsection{Moments}

If $Y_{m}$ is an Exp-G distribution with power parameter $m$, then we obtain the $r^{\text {th }}$ moment of the OW-TL-GPS family of distributions from equation (2.2) as follows:

$$
E\left(X^{r}\right)=\sum_{m=0}^{\infty} \phi_{m} E\left(Y_{m}^{r}\right),
$$

where $E\left(Y_{m}^{r}\right)$ is the $r^{\text {th }}$ moment of $Y_{m}$ which follows an Exp-G distribution with power parameter $m$ and $\phi_{m}$ is given by equation (2.3). The incomplete moments are obtained from the equation

$$
\mathrm{I}_{X}(\mathrm{t})=\int_{0}^{t} x^{r} f_{\theta}(x ; \xi) \mathrm{d} x=\sum_{m=0}^{\infty} \phi_{m} \mathrm{I}_{m}(t),
$$

where $I_{m}(t)=\int_{0}^{t} x^{s} g_{m}(x ; \xi) d x$ is the incomplete moment of the Exp-G distribution and $\phi_{m}$ is given by equation (2.3). The moment generating function (mgf) of $X$ is given by

$$
M_{X}(t)=\sum_{m=0}^{\infty} \phi_{m} E\left(e^{t Y_{m}}\right),
$$

where $E\left(e^{t \gamma_{m}}\right)$ is the mgf of the Exp-G distribution and $\phi_{m}$ is as defined in equation (2.3). The characteristic function and is given by $\psi(t)=E\left(e^{i t X}\right)$, where $i=\sqrt{-1}$, that is

$$
\psi(t)=\sum_{m=0}^{\infty} \phi_{\mathfrak{m}} \psi_{\mathfrak{m}}(t),
$$


where $\psi_{m}(t)$ is the characteristic function of Exp-G distribution and $\phi_{m}$ is as defined in equation (2.3).

We can readily obtain the following measures: coefficient of skewness (CS), coefficient of variation (CV), coefficient of kurtosis $(\mathrm{CK})$, variance $\left(\sigma^{2}\right)$, and standard deviation $(\mathrm{SD}=\sigma)$. Mathematically, $\mathrm{CS}=\frac{\mathrm{E}\left[(\mathrm{X}-\mu)^{3}\right]}{\left[\mathrm{E}(\mathrm{X}-\mu)^{2}\right]^{3 / 2}}=$ $\frac{\mu_{3}^{\prime}-3 \mu \mu_{2}^{\prime}+2 \mu^{3}}{\left(\mu_{2}^{\prime}-\mu^{2}\right)^{3 / 2}}, C V=\frac{\sigma}{\mu}=\frac{\sqrt{\mu_{2}^{\prime}-\mu^{2}}}{\mu}=\sqrt{\frac{\mu_{2}^{\prime}}{\mu^{2}}-1}, C K=\frac{E\left[(X-\mu)^{4}\right]}{\left[E(X-\mu)^{2}\right]^{2}}=\frac{\mu_{4}^{\prime}-4 \mu \mu_{3}^{\prime}+6 \mu^{2} \mu_{2}^{\prime}-3 \mu^{4}}{\left(\mu_{2}^{\prime}-\mu^{2}\right)^{2}}$, and $\sigma^{2}=\mu_{2}^{\prime}-\mu^{2}$, respectively. The $r^{\text {th }}$ cumulant of the random variable $X$ is obtained from the recursive relationship: $\kappa_{r}=\mu_{r}^{\prime}-$ $\sum_{s=1}^{r-1}\left(\begin{array}{c}r-1 \\ s-1\end{array}\right) \mu_{r-s}^{\prime} \kappa_{s}$, where $\mu_{r}^{\prime}=E\left(X-\mu_{1}^{\prime}\right)^{r}$, so that the CS and CK are given by $\gamma_{1}=\frac{\kappa_{3}}{\kappa_{2}^{3 / 2}}$ and $\gamma_{2}=\frac{\kappa_{4}}{\kappa_{2}^{2}}$. We present moments, SD, CV, CS, and CK for selected parameter values for the OW-TL-LLoGP distribution in Table 4.

Table 4: Moments of the OW-TL-LLoGP distribution for some parameters values.

\begin{tabular}{l|ccccc}
\hline & $(0.5,1.5,0.5,1.5)$ & $(0.5,1,0.5,1.2)$ & $(1.1,2.5,0.5,2.5)$ & $(0.5,1.5,0.5,0.5)$ & $(1.1,0.9,1,1.1)$ \\
\hline$E(X)$ & 0.2269 & 0.1705 & 0.6729 & 0.0265 & 0.2913 \\
$E\left(X^{2}\right)$ & 0.0709 & 0.0592 & 0.4650 & 0.0026 & 0.1458 \\
$E\left(X^{3}\right)$ & 0.0265 & 0.0279 & 0.3288 & 0.0004 & 0.0913 \\
$E\left(X^{4}\right)$ & 0.0112 & 0.0158 & 0.2370 & 0.0001 & 0.0646 \\
$E\left(X^{5}\right)$ & 0.0052 & 0.0100 & 0.1738 & 0.0000 & 0.0493 \\
$S D$ & 0.1395 & 0.1736 & 0.1109 & 0.0434 & 0.2470 \\
$C V$ & 0.6148 & 1.0181 & 0.1647 & 1.6373 & 0.8480 \\
$C S$ & 0.5933 & 1.4377 & -0.5081 & 3.3610 & 0.8792 \\
$C K$ & 2.8968 & 4.9772 & 3.2482 & 19.9252 & 2.9314 \\
\hline
\end{tabular}

\subsection{Mean deviation, Lorenz, and Bonferroni curves}

Let $X \sim$ OW-TL-LLoGPS $(\alpha, \gamma, \theta, \xi)$, the mean deviation about the mean and about the median are defined by

$$
\delta_{1}(x)=\int_{0}^{\infty}|x-\mu| f_{\theta}(x) d x \text { and } \delta_{2}(x)=\int_{0}^{\infty}|x-M| f_{\theta}(x) d x,
$$

respectively, where $\mu=E(X)$ and $M=\operatorname{Median}(X)$. The deviations can also be expressed as

$$
\delta_{1}(x)=2 \mu F_{\theta}(\mu)-2 \int_{0}^{\mu} x f_{\theta}(x) d x=2 \mu F_{\theta}(\mu)-2 \sum_{m=0}^{\infty} \phi_{m} I_{m}^{*}(t),
$$

and

$$
\delta_{2}(x)=\mu-2 \int_{0}^{M} x f_{\theta}(x) d x=\mu-\sum_{m=0}^{\infty} \phi_{m} I_{m}^{*}(t) .
$$

Bonferroni and Lorenz curves are given by

$$
B(p)=\frac{1}{p \mu} \int_{0}^{t} \sum_{m=0}^{\infty} x \phi_{m} g_{m}(x ; \xi) d x=\frac{1}{p \mu} \sum_{m=0}^{\infty} \phi_{m} I_{m}^{*}(t),
$$

and

$$
\mathrm{L}(\mathrm{p})=\frac{1}{\mu} \int_{0}^{\mathrm{t}} \sum_{\mathrm{m}=0}^{\infty} x \phi_{\mathfrak{m}} g_{\mathfrak{m}}(x ; \xi) \mathrm{d} x=\frac{1}{\mu} \sum_{m=0}^{\infty} \phi_{\mathfrak{m}} I_{m}^{*}(t),
$$

where $I_{m}^{*}(t)=\int_{0}^{t} x g_{m}(x ; \xi) d x$, is the first incomplete moment of the Exp-G distribution and $\phi_{m}$ is as given in equation (2.3).

\subsection{Distribution of order statistics}

We obtain the pdf of the $i^{\text {th }}$ order statistics from the OW-TL-GPS family of distributions using the equation

$$
f_{i: n}(x)=\frac{f_{\theta}(x)}{B(i, n-i+1)} \sum_{j=0}^{n-j}\left(\begin{array}{c}
n-i \\
j
\end{array}\right) F_{\theta}(x)^{j+i-1},
$$


where $B(.,$.$) is the beta function. Substituting the cdf of the OW-TL-GPS family of distributions, we have$

$$
f_{i: n}(x)=\frac{f(x)}{B(i, n-i+1)} \sum_{j=0}^{n-j}\left(\begin{array}{c}
n-i \\
j
\end{array}\right)\left(1-\frac{C\left(\theta\left(\exp \left\{-\left[\frac{\left[1-\bar{G}^{2}(x ; \xi)\right]^{\gamma}}{\left[1-\left(1-\bar{G}^{2}(x ; \xi)\right)^{\gamma}\right]}\right]^{\alpha}\right\}\right)\right)}{C(\theta)}\right)^{j+i-1} .
$$

Considering the expansion

$$
\begin{aligned}
& \left(1-\frac{C\left(\theta\left(\exp \left\{-\left[\frac{\left[1-\bar{G}^{2}(x ; \xi)\right]^{\gamma}}{\left[1-\left(1-\overline{\mathrm{G}}^{2}(x ; \xi)\right)^{\gamma}\right]}\right]^{\alpha}\right\}\right)\right)}{C(\theta)}\right)^{j+i-1}=\sum_{l=0}^{j+i-1}(-1)^{\mathrm{l}}\left(\begin{array}{c}
j+i-1 \\
l
\end{array}\right) \\
& \times\left(\frac{C\left(\theta\left(\exp \left\{-\left[\frac{\left[1-\bar{G}^{2}(x ; \xi)\right]^{\gamma}}{\left[1-\left(1-\overline{\mathrm{G}}^{2}(x ; \xi)\right)^{\gamma}\right]}\right]^{\alpha}\right\}\right)\right)}{C(\theta)}\right)^{l}
\end{aligned}
$$

yields

$$
f_{i: n}(x)=\frac{f_{\theta}(x)}{B(i, n-i+1)} \sum_{l=0}^{j+i-1} \sum_{j=0}^{n-j}(-1)^{l}\left(\begin{array}{c}
n-i \\
j
\end{array}\right)\left(\begin{array}{c}
j+i-1 \\
l
\end{array}\right)\left(\frac{C\left(\theta\left(\exp \left\{-\left[\frac{\left[1-\bar{G}^{2}(x ; \xi)\right]^{\gamma}}{\left[1-\left(1-\bar{G}^{2}(x ; \xi)\right)^{\gamma}\right]}\right]^{\alpha}\right\}\right)\right)}{C(\theta)}\right)^{l}
$$

which simplifies to

$$
\begin{aligned}
f_{i: n}(x)= & \frac{f_{\theta}(x)}{B(i, n-i+1)} \sum_{l=0}^{j+i-1} \sum_{j=0}^{n-j} \frac{(-1)^{l+z}}{C^{l}(\theta)}\left(\begin{array}{c}
n-i \\
j
\end{array}\right)\left(\begin{array}{c}
j+i-1 \\
l
\end{array}\right) \\
& \times \sum_{z=0}^{\infty} d_{z, l} \theta^{z} \exp \left\{-z\left[\frac{\left[1-\bar{G}^{2}(x ; \xi)\right]^{\gamma}}{\left[1-\left(1-\bar{G}^{2}(x ; \xi)\right)^{\gamma}\right]}\right]^{\alpha}\right\},
\end{aligned}
$$

by applying the power series raised to a positive integer by [17], where $d_{z, l}=\left(z b_{0}\right)^{-1} \sum_{h=1}^{z}[l(h+1)-$ $z] b_{h} d_{z-h, l}$ and $d_{0, l}=b_{0}^{l}$. Furthermore, applying the following expansions

$$
\begin{aligned}
\exp \left\{-z\left[\frac{\left[1-\overline{\mathrm{G}}^{2}(x ; \xi)\right]^{\gamma}}{\left[1-\left(1-\overline{\mathrm{G}}^{2}(x ; \xi)\right)^{\gamma}\right]}\right]^{\alpha}\right\} & =\sum_{p=0}^{\infty} \frac{(-1)^{\mathrm{p}} z^{\mathrm{p}}}{\mathrm{p} !} \frac{\left(1-\overline{\mathrm{G}}^{2}(x ; \xi)\right)^{\gamma \alpha p}}{\left[1-\left(1-\overline{\mathrm{G}}^{2}(x ; \xi)\right)^{\gamma}\right]^{\alpha p}} \\
{\left[1-\left(1-\overline{\mathrm{G}}^{2}(x ; \xi)\right)^{\gamma}\right]^{-\alpha p} } & =\sum_{s=0}^{\infty}(-1)^{s}\left(\begin{array}{c}
-\alpha \mathrm{p} \\
s
\end{array}\right)\left(1-\overline{\mathrm{G}}^{2}(x ; \xi)\right)^{\gamma s} \\
\left(1-\overline{\mathrm{G}}^{2}(x ; \xi)\right)^{\gamma(\alpha \mathrm{p}+\mathrm{s})} & =\sum_{\mathrm{r}=0}^{\infty}(-1)^{\mathrm{r}}\left(\begin{array}{c}
\gamma(\alpha \mathrm{p}+\mathrm{s}) \\
\mathrm{r}
\end{array}\right) \overline{\mathrm{G}}^{2 \mathrm{r}}(x ; \xi)
\end{aligned}
$$

and

$$
\overline{\mathrm{G}}^{2 \mathrm{r}}(x ; \xi)=\sum_{\mathrm{q}=0}^{\infty}(-1)^{\mathrm{q}}\left(\begin{array}{c}
2 \mathrm{r} \\
\mathrm{q}
\end{array}\right) \mathrm{G}^{\mathrm{q}}(x ; \xi),
$$

we get

$$
f_{i: n}(x)=\frac{1}{B(i, n-i+1)} \sum_{z, p, s, r, q}^{\infty} \sum_{l=0}^{j+i-1} \sum_{j=0}^{n-j} \frac{(-1)^{l+z+p+s+r+q} z^{p} \theta^{z}}{p ! C^{l}(\theta)} d_{z, l}\left(\begin{array}{c}
n-i \\
j
\end{array}\right)\left(\begin{array}{c}
j+i-1 \\
l
\end{array}\right)
$$




$$
\times\left(\begin{array}{c}
-\alpha p \\
s
\end{array}\right)\left(\begin{array}{c}
\gamma(\alpha p+s) \\
r
\end{array}\right)\left(\begin{array}{c}
2 r \\
q
\end{array}\right) f_{\theta}(x) G^{q}(x ; \xi) .
$$

Using results in equation (2.2) for $f_{\theta}(x)$, we get

$$
\begin{aligned}
f_{i: n}(x)= & \frac{1}{B(i, n-i+1)} \sum_{z, p, s, r, q} \sum_{m=0}^{\infty} \sum_{l=0}^{j+i-1} \sum_{j=0}^{n-j} \frac{(-1)^{l+z+p+s+r+q} z^{p} \theta^{z}}{p ! C^{l}(\theta)} d_{z, l} \phi_{m}\left(\begin{array}{c}
n-i \\
j
\end{array}\right)\left(\begin{array}{c}
j+i-1 \\
l
\end{array}\right) \\
& \times\left(\begin{array}{c}
-\alpha p \\
s
\end{array}\right)\left(\begin{array}{c}
\gamma(\alpha p+s) \\
r
\end{array}\right)\left(\begin{array}{c}
2 r \\
q
\end{array}\right)(m+1) g(x ; \xi) G^{q+m}(x ; \xi)=\sum_{q, m=0}^{\infty} \phi_{q, m}^{*} g_{q+m}(x ; \xi),
\end{aligned}
$$

where

$$
\begin{aligned}
\phi_{q, m}^{*}= & \frac{1}{B(i, n-i+1)} \sum_{z, p, s, r}^{\infty} \sum_{l=0}^{j+i-1} \sum_{j=0}^{n-j} \frac{(-1)^{l+z+p+s+r+q} z^{p} \theta^{z}}{p ! C^{l}(\theta)} d_{z, l} \phi_{m}\left(\begin{array}{c}
n-i \\
j
\end{array}\right)\left(\begin{array}{c}
j+i-1 \\
l
\end{array}\right) \\
& \times\left(\begin{array}{c}
-\alpha p \\
s
\end{array}\right)\left(\begin{array}{c}
\gamma(\alpha p+s) \\
r
\end{array}\right)\left(\begin{array}{c}
2 r \\
q
\end{array}\right) \frac{m+1}{q+m+1}
\end{aligned}
$$

and $g_{q+m}(x ; \xi)=(q+m+1) g(x ; \xi) G^{q+m}(x ; \xi)$ is the Exp-G distribution with power parameter $(q+m)$.

\subsection{Rényi entropy}

Entropy is a measure of variation of uncertainty for a random variable $X$ with $\operatorname{pdf} f(x)$. There are two popular measures of entropy, namely Shannon entropy and Rényi entropy. Shannon entropy is due to [29] and Rényi entropy is due to [28]. Rényi entropy is defined by

$$
I_{R}(v)=(1-v)^{-1} \log \left[\int_{0}^{\infty} f_{\theta}^{v}(x) d x\right], v \neq 1, v>0,
$$

and Shannon entropy is given by $E\{-\log [f(X)]\}$. Shannon entropy is a special case of Rényi entropy, we therefore, derive expressions for Rényi entropy for the OW-TL-GPS distribution. Substituting the pdf of the OW-TL-GPS family of distributions, we have $f^{\vee}(x)$ given by

$$
\begin{aligned}
f_{\theta}^{\nu}(x)= & \frac{(2 \theta \gamma \alpha)^{v} g^{\nu}(x ; \xi) \bar{G}^{v}(x ; \xi)\left[1-\bar{G}^{2}(x ; \xi)\right]^{(\gamma \alpha-1) v}}{\left[1-\left(1-\bar{G}^{2}(x ; \xi)\right)^{\gamma}\right]^{(\alpha+1) \nu}} \exp \left\{-v\left[\frac{\left[1-\bar{G}^{2}(x ; \xi)\right]^{\gamma}}{\left[1-\left(1-\bar{G}^{2}(x ; \xi)\right)^{\gamma}\right]}\right]^{\alpha}\right\} \\
& \times\left(\frac{C^{\prime}\left(\theta\left(\exp \left\{-\left[\frac{\left[1-\bar{G}^{2}(x ; \xi)\right]^{\gamma}}{\left[1-\left(1-\bar{G}^{2}(x ; \xi)\right)^{\gamma}\right]}\right]^{\alpha}\right\}\right)\right)}{C(\theta)}\right)^{v} \cdot
\end{aligned}
$$

By using the power series raised to a positive integer by [17], where $d_{z, l}=\left(z b_{0}\right)^{-1} \sum_{h=1}^{z}[l(h+1)-$ $z] b_{h} d_{z-h, l}$ and $d_{0, l}=b_{0}^{l}$, we get

$$
\begin{aligned}
f_{\theta}^{\nu}(x)= & \frac{(2 \theta \gamma \alpha)^{v} g^{\nu}(x ; \xi) \bar{G}^{\nu}(x ; \xi)\left[1-\bar{G}^{2}(x ; \xi)\right]^{(\gamma \alpha-1) v}}{C^{v}(\theta)\left[1-\left(1-\bar{G}^{2}(x ; \xi)\right)^{\gamma}(\alpha+1) v\right.} \exp \left\{-v\left[\frac{\left[1-\bar{G}^{2}(x ; \xi)\right]^{\gamma}}{\left[1-\left(1-\bar{G}^{2}(x ; \xi)\right)^{\gamma}\right]}\right]^{\alpha}\right\} \\
& \times \sum_{z=0}^{\infty} d_{z, l} \theta^{z} \exp \left\{-z\left[\frac{\left[1-\bar{G}^{2}(x ; \xi)\right]^{\gamma}}{\left[1-\left(1-\bar{G}^{2}(x ; \xi)\right)^{\gamma}\right]}\right]^{\alpha}\right\} .
\end{aligned}
$$

Considering the following expansions

$$
\exp \left\{-(z+v)\left[\frac{\left[1-\overline{\mathrm{G}}^{2}(x ; \xi)\right]^{\gamma}}{\left[1-\left(1-\overline{\mathrm{G}}^{2}(x ; \xi)\right)^{\gamma}\right]}\right]^{\alpha}\right\}=\sum_{\mathrm{p}=0}^{\infty} \frac{(-1)^{\mathrm{p}}(z+v)^{\mathrm{p}}}{\mathrm{p} !}\left[\frac{\left[1-\overline{\mathrm{G}}^{2}(x ; \xi)\right]^{\gamma \alpha p}}{\left[1-\left(1-\overline{\mathrm{G}}^{2}(x ; \xi)\right)^{\gamma}\right] \alpha \mathrm{p}}\right],
$$




$$
\begin{aligned}
{\left[1-\left(1-\overline{\mathrm{G}}^{2}(x ; \xi)\right)^{\gamma}\right]^{-(\alpha(p+v)+v)} } & =\sum_{s=0}^{\infty}(-1)^{s}\left(\begin{array}{c}
-(\alpha(p+v)+v) \\
s
\end{array}\right)\left(1-\bar{G}^{2}(x ; \xi)\right)^{\gamma s}, \\
\left(1-\overline{\mathrm{G}}^{2}(x ; \xi)\right)^{\alpha \gamma(p+v)+\gamma s-v} & =\sum_{r=0}^{\infty}(-1)^{r}\left(\begin{array}{c}
\alpha \gamma(p+v)+\gamma s-v \\
r
\end{array}\right) \bar{G}(x ; \xi)^{2 r}
\end{aligned}
$$

and

$$
\overline{\mathrm{G}}(x ; \xi)^{2 \mathrm{r}+v}=\sum_{\mathrm{m}=0}^{\infty}(-1)^{\mathrm{m}}\left(\begin{array}{c}
2 \mathrm{r}+v \\
\mathrm{~m}
\end{array}\right) \mathrm{G}^{\mathrm{m}}(x ; \xi),
$$

yields

$$
\begin{aligned}
f_{\theta}^{v}(x)= & \sum_{z, p, s, r, m=0}^{\infty} \frac{(2 \gamma \alpha)^{v} \theta^{v+z}(-1)^{p+s+r+m}(z+v)^{p} d_{z, v}}{C^{v}(\theta) p !}\left(\begin{array}{c}
-(\alpha(p+v)+v) \\
s
\end{array}\right) \\
& \times\left(\begin{array}{c}
\alpha \gamma(p+v)+\gamma s-v \\
r
\end{array}\right)\left(\begin{array}{c}
2 r+v \\
m
\end{array}\right) g^{v}(x ; \xi) G^{m}(x ; \xi) .
\end{aligned}
$$

Therefore, the Rényi entropy of the OW-TL-GPS family of distributions is given by

$$
\mathrm{I}_{\mathrm{R}}(v)=(1-v)^{-1} \log \left[\sum_{\mathrm{m}=0}^{\infty} \psi_{\mathrm{m}} e^{(1-v) \mathrm{I}_{\mathrm{REG}}}\right],
$$

where

$$
\begin{aligned}
\psi_{m}= & \sum_{z, p, s, r}^{\infty} \frac{(2 \gamma \alpha)^{v} \theta^{v+z}(-1)^{p+s+r+m}(z+v)^{p} d_{z, v}}{C^{v}(\theta) p !}\left(\begin{array}{c}
-(\alpha(p+v)+v) \\
s
\end{array}\right)\left(\begin{array}{c}
\alpha \gamma(p+v)+\gamma s-v \\
r
\end{array}\right) \\
& \times\left(\begin{array}{c}
2 r+v \\
m
\end{array}\right) \frac{1}{(m / v+1)^{v}}
\end{aligned}
$$

and $I_{R E G}=\int_{0}^{\infty}\left[\left(\frac{m}{v}+1\right) g(x ; \xi)[G(x ; \xi)]^{\frac{m}{v}}\right]^{v} d x$ is Rényi entropy of Exp-G distribution with parameter $\frac{m}{v}$.

\section{Maximum likelihood estimation}

Let $X_{i} \sim \mathrm{OW}-\mathrm{TL}-\mathrm{GPS}(\alpha, \gamma, \theta ; \xi)$ and $\Phi=(\alpha, \gamma, \theta ; \xi)^{\top}$ be the parameter vector. The log-likelihood $\ell=\ell(\Phi)$ based on a random sample of size $\mathrm{n}$ is given by

$$
\begin{aligned}
\ell=\ell(\Phi)= & n \log (2 \theta \gamma \alpha)+\sum_{n=1}^{n} \log \left[g\left(x_{i} ; \xi\right)\right]+\sum_{i=1}^{n} \log \left[\bar{G}\left(x_{i} ; \xi\right)\right]+(\gamma \alpha-1) \sum_{i=1}^{n} \log \left[1-\bar{G}^{2}\left(x_{i} ; \xi\right)\right] \\
& -(\alpha+1) \sum_{i=1}^{n} \log \left[1-\left(1-\bar{G}^{2}\left(x_{i} ; \xi\right)\right)^{\gamma}\right]-\sum_{i=1}^{n} t-\sum_{i=1}^{n} \log [C(\theta)]+\sum_{i=1}^{n} \log \left[C^{\prime}(\theta(\exp (-t)))\right] .
\end{aligned}
$$

The elements of the score vector $\mathrm{U}=\left(\frac{\partial \ell}{\partial \alpha}, \frac{\partial \ell}{\partial \gamma}, \frac{\partial \ell}{\partial \theta}, \frac{\partial \ell}{\partial \xi_{k}}\right)$ are

$$
\begin{aligned}
\frac{\partial \ell}{\partial \alpha}= & \frac{n}{\alpha}+\gamma \sum_{i=1}^{n} \log [t] \ln \left[\frac{\left(1-\overline{\mathrm{G}}^{2}\left(x_{i} ; \xi\right)\right)^{\gamma}}{\left[1-\left(1-\overline{\mathrm{G}}^{2}\left(x_{i} ; \xi\right)\right)^{\gamma}\right]}\right]-\sum_{i=1}^{n} \log \left[1-\left(1-\overline{\mathrm{G}}^{2}\left(x_{i} ; \xi\right)\right)^{\gamma}\right] \\
& -\sum_{i=1}^{n} \frac{\mathrm{C}^{\prime \prime}(\theta(\exp (-\mathrm{t})))}{\mathrm{C}^{\prime}(\theta(\exp (-\mathrm{t})))} \theta(\exp (-\mathrm{t})) \mathrm{t} \log \left[\frac{\left(1-\overline{\mathrm{G}}^{2}\left(x_{i} ; \xi\right)\right)^{\gamma}}{1-\left(1-\overline{\mathrm{G}}^{2}\left(x_{i} ; \xi\right)\right)^{\gamma}}\right], \\
\frac{\partial \ell}{\partial \gamma}= & \frac{n}{\gamma}+\alpha \sum_{i=1}^{n} \log \left[1-\overline{\mathrm{G}}^{2}\left(x_{i} ; \xi\right)\right]+(\alpha+1) \sum_{i=1}^{n} \frac{\left(1-\overline{\mathrm{G}}^{2}\left(x_{i} ; \xi\right)\right)^{\gamma} \log \left[1-\overline{\mathrm{G}}^{2}\left(x_{i} ; \xi\right)\right]}{1-\left(1-\overline{\mathrm{G}}^{2}\left(x_{i} ; \xi\right)\right)^{\gamma}}
\end{aligned}
$$




$$
\begin{aligned}
& -\sum_{i=1}^{n} \frac{C^{\prime \prime}(\theta(\exp (-t)))}{C^{\prime}(\theta(\exp (-t)))} \theta(\exp (-t)) \frac{\log \left[1-\bar{G}^{2}\left(x_{i} ; \xi\right)\right]\left(1-\bar{G}^{2}\left(x_{i} ; \xi\right)\right)^{\gamma}\left(1-\bar{G}^{2}\left(x_{i} ; \xi\right)\right)^{\gamma \alpha+\gamma-1}}{\left(1-\left(1-\bar{G}^{2}\left(x_{i} ; \xi\right)\right)^{\gamma}\right)^{\alpha+1}}, \\
\frac{\partial \ell}{\partial \theta}= & \frac{n}{\theta}+\sum_{i=1}^{n} \frac{C^{\prime \prime}(\theta(\exp (-t)))}{C^{\prime}(\theta(\exp (-t)))} \exp (-t)+n \frac{C^{\prime}(\theta)}{C(\theta)}, \\
\frac{\partial \ell}{\partial \xi_{k}}= & \sum_{i=1}^{n} \frac{1}{g\left(x_{i} ; \xi\right)} \frac{\partial g\left(x_{i} ; \xi\right)}{\partial \xi_{k}}+(\gamma \alpha-1) \sum_{i=1}^{n} \frac{1}{\left(1-\bar{G}^{2}\left(x_{i} ; \xi\right)\right)^{\gamma}} \frac{\partial\left(1-\bar{G}^{2}\left(x_{i} ; \xi\right)\right)^{\gamma}}{\partial \xi_{k}} \\
& +\sum_{i=0}^{n} \frac{1}{\bar{G}\left(x_{i} ; \xi\right)} \frac{\partial G\left(x_{i} ; \xi\right)}{\partial \xi_{k}}-(\alpha+1) \sum_{i=1}^{n} \frac{1}{\left(1-\left(1-\bar{G}^{2}\left(x_{i} ; \xi\right)\right)^{\gamma}\right)} \frac{\partial\left(1-\left(1-\bar{G}^{2}\left(x_{i} ; \xi\right)\right)^{\gamma}\right)}{\partial \xi_{k}} \\
& -\sum_{i=1}^{n} \frac{\left(1-\left(1-\bar{G}^{2}\left(x_{i} ; \xi\right)\right)^{\gamma}\right) \frac{\partial\left(1-\bar{G}^{2}\left(x_{i} ; \xi\right)\right)^{\gamma}}{\partial \xi_{k}}-\left(1-\bar{G}^{2}\left(x_{i} ; \xi\right)\right)^{\gamma} \frac{\partial\left(1-\left(1-\bar{G}^{2}\left(x_{i} ; \xi\right)\right)^{\gamma}\right)}{\partial \xi_{k}}}{\left(1-\left(1-\bar{G}^{2}\left(x_{i} ; \xi\right)\right)^{\gamma}\right)^{2}} \\
& +\sum_{i=1}^{n} \frac{1}{C^{\prime}(\theta(\exp (-t)))} \frac{\partial C^{\prime}(\theta(\exp (-t)))}{\partial \xi_{k}},
\end{aligned}
$$

respectively. The maximum likelihood estimates of the parameters, denoted by $\hat{\Phi}$ is obtained by solving the nonlinear equation $\left(\frac{\partial \ell}{\partial \alpha}, \frac{\partial \ell}{\partial \gamma}, \frac{\partial \ell}{\partial \theta}, \frac{\partial \ell}{\partial \xi_{k}}\right)^{\top}=0$, using a numerical method such as Newton-Raphson procedure. The Fisher information matrix is given by $\mathbf{I}(\Phi)=\left[\mathbf{I}_{\theta_{i}, \theta_{j}}\right]_{(3+q) \times(3+q)}=E\left(-\frac{\partial^{2} \ell}{\partial \theta_{i} \partial \theta_{j}}\right), i, j=$ $1,2, \ldots, 3+q$ can be numerically obtained by using MATLAB or NLMIXED in SAS or $R$ software. The total Fisher information matrix $n \mathbf{I}(\Phi)$ can be approximated by

$$
\mathbf{J}_{\mathfrak{n}}(\hat{\Phi}) \approx\left[-\left.\frac{\partial^{2} \ell}{\partial \theta_{i} \partial \theta_{j}}\right|_{\Phi}=\hat{\Phi}\right]_{(3+q) \times(3+q)}, \quad i, j=1,2, \ldots, 3+q,
$$

where $\mathrm{q}$ is the number of components in the vector of parameters $\xi$. Note that for a given set of observations, the matrix given in equation (3.1) is obtained after the convergence of the Newton-Raphson procedure via NLMIXED in SAS or R software.

\section{Some special cases}

We consider some special cases when the baseline distribution is log-logistic distribution. The cdf and pdf of the $\log$ logistic distribution are given by $G(x ; \lambda)=1-\left(1+x^{\lambda}\right)^{-1}$ and $g(x ; \lambda)=\lambda x^{\lambda-1}\left(1+x^{\lambda}\right)^{-2}$, for $\lambda>0$.

\subsection{The odd Weibull-Topp-Leone-log-logistic power series distribution}

We present the odd Weibull-Topp-Leone-log-logistic power series (OW-TL-LLoGPS) distribution. The OW-TL-LLoGPS distribution has its cdf and pdf defined by

$$
F_{\theta}(x)=1-\frac{C\left(\theta\left(\exp \left\{-\left[\frac{\left[1-\left(1+x^{\lambda}\right)^{-2}\right]^{\gamma}}{\left[1-\left(1-\left(1+x^{\lambda}\right)^{-2}\right)^{\gamma}\right]}\right]^{\alpha}\right\}\right)\right)}{C(\theta)}
$$

and

$$
\begin{aligned}
f_{\theta}(x)= & \frac{2 \gamma \alpha \lambda x^{\lambda-1}\left(1+\chi^{\lambda}\right)^{-3}\left[1-\left(1+x^{\lambda}\right)^{-2}\right]^{\gamma \alpha-1}}{\left[1-\left(1-\left(1+x^{\lambda}\right)^{-2}\right)^{\gamma}\right]^{\alpha+1}} \exp \left\{-\left[\frac{\left[1-\left(1+x^{\lambda}\right)^{-2}\right]^{\gamma}}{\left[1-\left(1-\left(1+x^{\lambda}\right)^{-2}\right)^{\gamma}\right]}\right]^{\alpha}\right\} \\
& \times \frac{C^{\prime}\left(\theta\left(\exp \left\{-\left[\frac{\left[1-\left(1+x^{\lambda}\right)^{-2}\right]^{\gamma}}{\left[1-\left(1-\left(1+x^{\lambda}\right)^{-2}\right)^{\gamma}\right]}\right]^{\alpha}\right\}\right)\right)}{C(\theta)},
\end{aligned}
$$

respectively, for $\alpha, \lambda, \gamma, \theta>0$. 


\subsubsection{Sub-models of the OW-TL-LLoGPS distribution}

We present three sub-models of the OW-TL-LLoGPS distribution, namely, OW-TL-LLoG Poisson (OWTL-LLoGP), OW-TL-LLoG logarithmic (OW-TL-LLoGL) and OW-TL-LLoG geometric (OW-TL-LLoGG) distributions in this section.

- OW-TL-LLoGP distribution: The OW-TL-LLoGP distribution has its cdf and pdf given by

$$
\mathrm{F}_{\mathrm{OW}-\mathrm{TL}-\mathrm{LLoGP}}(x ; \alpha, \lambda, \gamma, \theta)=1-\frac{\exp \left(\theta\left(\exp \left\{-\left[\frac{\left[1-\left(1+\mathrm{x}^{\lambda}\right)^{-2}\right]^{\gamma}}{\left[1-\left(1-\left(1+\mathrm{x}^{\lambda}\right)^{-2}\right)^{\gamma}\right]}\right]^{\alpha}\right\}\right)\right)-1}{\exp (\theta)-1}
$$

and

$$
\begin{aligned}
f_{\text {OW-TL-LLoGP }}(x ; \alpha, \lambda, \gamma, \theta)= & \frac{2 \theta \gamma \alpha \lambda x^{\lambda-1}\left(1+x^{\lambda}\right)^{-3}\left[1-\left(1+x^{\lambda}\right)^{-2}\right]^{\gamma \alpha-1}}{\left[1-\left(1-\left(1+x^{\lambda}\right)^{-2}\right)^{\gamma}\right]^{\alpha+1}} \\
& \times \exp \left\{-\left[\frac{\left[1-\left(1+x^{\lambda}\right)^{-2}\right]^{\gamma}}{\left[1-\left(1-\left(1+x^{\lambda}\right)^{-2}\right)^{\gamma}\right]}\right]^{\alpha}\right\} \\
& \times \frac{\exp \left(\theta\left(\exp \left\{-\left[\frac{\left[1-\left(1+x^{\lambda}\right)^{-2}\right]^{\gamma}}{\left[1-\left(1-\left(1+x^{\lambda}\right)^{-2}\right)^{\gamma}\right]}\right]^{\alpha}\right\}\right)\right)}{\exp (\theta)-1},
\end{aligned}
$$

respectively, for $\alpha, \lambda, \gamma, \theta>0$.
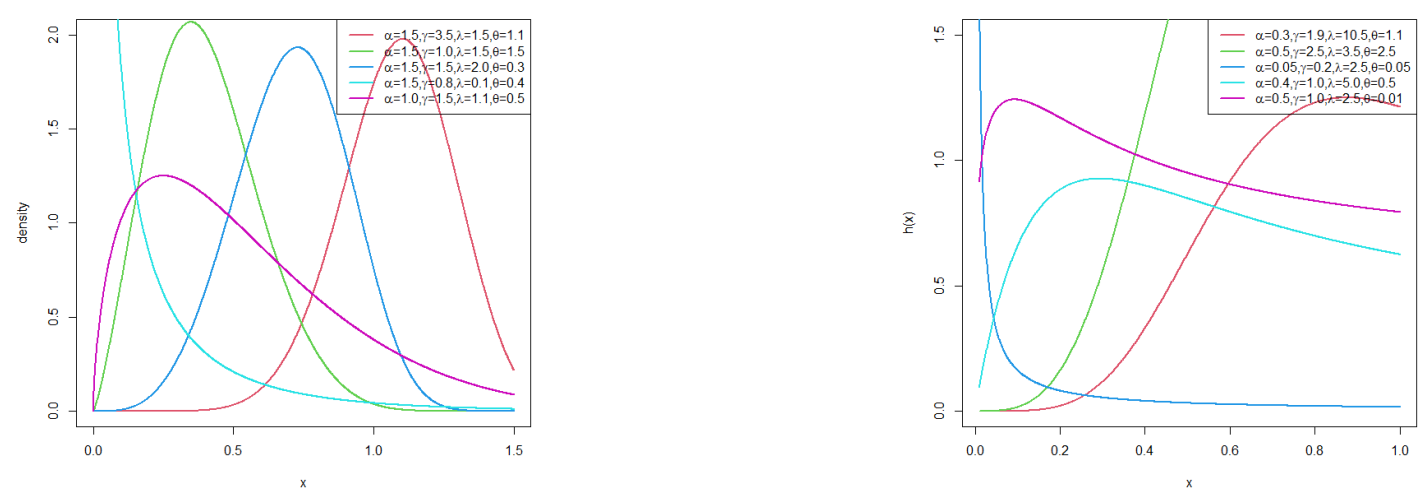

Figure 1: pdfs and hrfs plots for OW-TL-LLoGP distribution.

The pdf of the OW-TL-LLoGP distribution can handle data that is almost symmetric, reverse-J, left or right-skewed. Also, the hazard rate function of the distribution exhibit both monotonic and nonmonotonic shapes.

- OW-TL-LLoGL distribution: The cdf and pdf of the OW-TL-LLoGL distribution are given by

$$
\mathrm{F}_{\mathrm{OW}-\mathrm{TL}-\mathrm{LLoGL}}(x ; \alpha, \lambda, \gamma, \theta)=1-\frac{\log \left(1-\left(\theta\left(\exp \left\{-\left[\frac{\left[1-\left(1+\mathrm{x}^{\lambda}\right)^{-2} \gamma^{\gamma}\right.}{\left[1-\left(1-\left(1+\mathrm{x}^{\lambda}\right)^{-2}\right)^{\gamma}\right]}\right]^{\alpha}\right\}\right)\right)\right)}{\log (1-\theta)}
$$

and

$$
\begin{aligned}
f_{\text {OW-TL-LLoGL }}(x ; \alpha, \lambda, \gamma, \theta)= & \frac{2 \theta \gamma \alpha \lambda x^{\lambda-1}\left(1+x^{\lambda}\right)^{-3}\left[1-\left(1+x^{\lambda}\right)^{-2}\right]^{\gamma \alpha-1}}{\left[1-\left(1-\left(1+x^{\lambda}\right)^{-2}\right)^{\gamma}\right]^{\alpha+1}} \\
& \times \exp \left\{-\left[\frac{\left[1-\left(1+x^{\lambda}\right)^{-2}\right]^{\gamma}}{\left[1-\left(1-\left(1+x^{\lambda}\right)^{-2}\right)^{\gamma}\right]}\right]^{\alpha}\right\} \\
& \times \frac{\left(1-\left(\theta\left(\exp \left\{-\left[\frac{\left[1-\left(1+x^{\lambda}\right)^{-2}\right]^{\gamma}}{\left[1-\left(1-\left(1+x^{\lambda}\right)^{-2}\right)^{\gamma}\right]}\right]^{\alpha}\right\}\right)\right)\right)^{-1}}{-\log (1-\theta)}
\end{aligned}
$$


respectively, for $\alpha, \lambda, \gamma>0$ and $0<\theta<1$. The pdf of the OW-TL-LLoGL distribution can handle data that
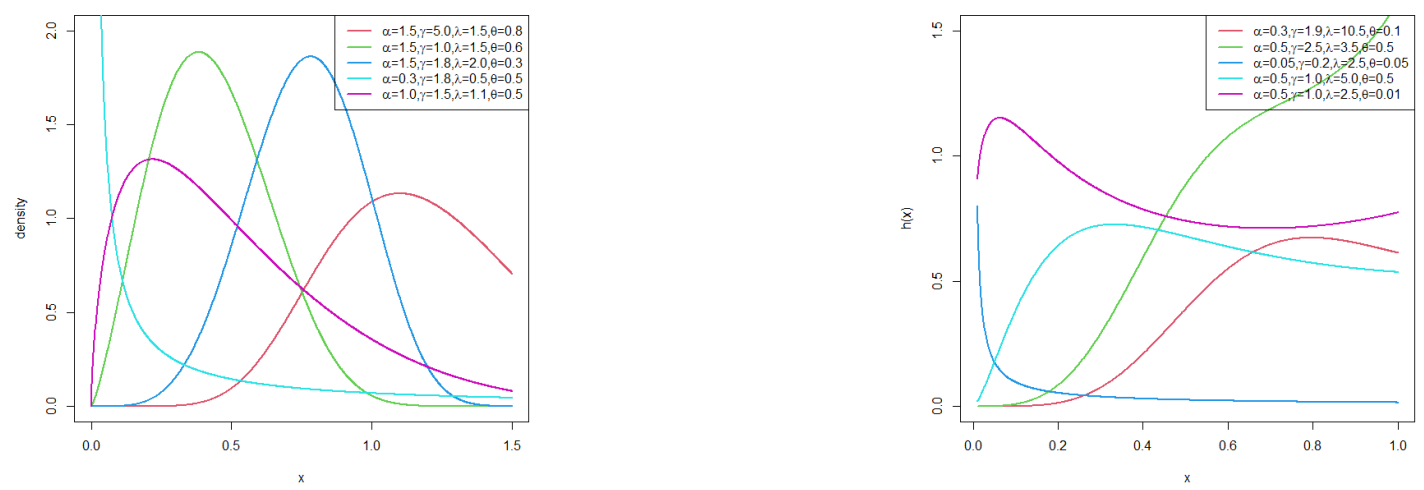

Figure 2: pdfs and hrfs plots for OW-TL-LLoGL distribution.

is almost symmetric, reverse-J, left or right-skewed. Also the hazard function of the distribution exhibit decreasing, increasing, uni-modal and upside bathtub followed by bathtub shapes.

- OW-TL-LLoGG distribution: The cdf and pdf of the OW-TL-LLoGG distribution are given by

$$
\mathrm{F}_{\mathrm{OW}-\mathrm{TL}-\mathrm{LLoGG}}(\mathrm{x} ; \alpha, \lambda, \gamma, \theta)=1-\frac{(1-\theta) \exp \left\{-\left[\frac{\left[1-\left(1+\mathrm{x}^{\lambda}\right)^{-2}\right]^{\gamma}}{\left[1-\left(1-\left(1+\mathrm{x}^{\lambda}\right)^{-2}\right)^{\gamma]}\right.}\right]^{\alpha}\right\}}{1-\theta \exp \left\{-\left[\frac{\left[1-\left(1+\mathrm{x}^{\lambda}\right)^{-2}\right]^{\gamma}}{\left[1-\left(1-\left(1+\mathrm{x}^{\lambda}\right)^{-2}\right)^{\gamma]}\right]}\right]^{\alpha}\right\}}
$$

and

$$
\begin{aligned}
f_{\mathrm{OW}-\mathrm{TL}-\mathrm{LLoGG}}(x ; \alpha, \lambda, \gamma, \theta)= & \frac{2(1-\theta) \gamma \alpha \lambda x^{\lambda-1}\left(1+x^{\lambda}\right)^{-3}\left[1-\left(1+x^{\lambda}\right)^{-2}\right]^{\gamma \alpha-1}}{\left[1-\left(1-\left(1+x^{\lambda}\right)^{-2}\right)^{\gamma}\right]^{\alpha+1}} \\
& \times \exp \left\{-\left[\frac{\left[1-\left(1+x^{\lambda}\right)^{-2}\right]^{\gamma}}{\left[1-\left(1-\left(1+x^{\lambda}\right)^{-2}\right)^{\gamma}\right]}\right]^{\alpha}\right\} \\
& \times\left(1-\left(\theta\left(\exp \left\{-\left[\frac{\left[1-\left(1+x^{\lambda}\right)^{-2}\right]^{\gamma}}{\left[1-\left(1-\left(1+x^{\lambda}\right)^{-2}\right)^{\gamma}\right]}\right]^{\alpha}\right\}\right)\right)\right)^{-2},
\end{aligned}
$$

respectively, for $\alpha, \lambda, \gamma>0$ and $0<\theta<1$.
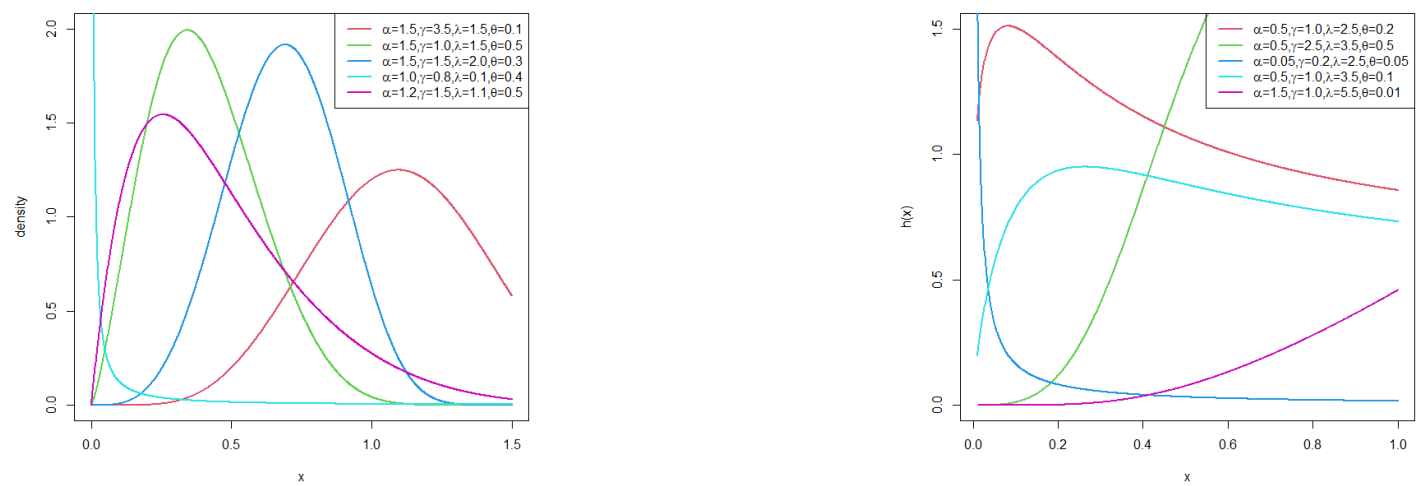

Figure 3: pdfs and hrfs plots for OW-TL-LLoGG distribution.

The pdf of the OW-TL-LLoGG distribution can handle data that is almost symmetric, reverse-J, left or right-skewed. Also the hazard function of the distribution exhibit reverse-J, J, increasing, uni-modal and upside bathtub shapes. 


\section{Simulation study}

In this section, a simulation study is contacted to examine the performance of the maximum likelihood estimates. We simulated 1000 samples for the following sets of parameters: I: $\alpha=1.5, \lambda=0.5, \gamma=$ $0.05, \theta=1.0$, II: $\alpha=1.5, \lambda=0.5, \gamma=1.5, \theta=1.0$, III: $\alpha=1.5, \lambda=0.5, \gamma=0.5, \theta=1.0$, IV: $\alpha=1.5, \lambda=$ 1.0, $\gamma=1.0, \theta=0.5, \mathrm{~V}: \alpha=1.5, \lambda=1.0, \gamma=0.2, \theta=0.5$ and VI: $\alpha=1.5, \lambda=1.0, \gamma=1.5, \theta=0.5$. We considered the sample sizes $(n=25,50,100,200,400,800)$. We calculated the mean, root mean square error (RMSE) and average bias. The bias and RMSE are given by the following formulae $\operatorname{Bias}(\hat{\theta})=\frac{\sum_{i=1}^{N} \hat{\theta}_{i}}{N}-\theta$, and $\operatorname{RMSE}(\hat{\theta})=\sqrt{\frac{\sum_{i=1}^{N}\left(\hat{\theta}_{i}-\theta\right)^{2}}{N}}$, respectively. We observe that as the sample size increases, the mean approximates the true parameters values, the RMSE and average bias decay toward zero for all parameters values.

Table 5: Monte Carlo simulation results for OW-TL-LLoGP distribution: mean, RMSE, and average bias.

\begin{tabular}{|c|c|c|c|c|c|c|c|c|c|c|}
\hline & & \multicolumn{3}{|c|}{ I: $\alpha=1.5, \lambda=0.5, \gamma=0.05, \theta=1.0$} & \multicolumn{3}{|c|}{ II: $\alpha=1.5, \lambda=0.5, \gamma=1.5, \theta=1.0$} & \multicolumn{3}{|c|}{ III: $\alpha=1.5, \lambda=0.5, \gamma=0.5, \theta=1.0$} \\
\hline & $\mathrm{n}$ & Mean & RMSE & Bias & Mean & RMSE & Bias & Mean & RMSE & Bias \\
\hline \multirow{6}{*}{$\alpha$} & 25 & 1.988135 & 0.851713 & 0.488135 & 1.855894 & 1.053127 & 0.355894 & 1.920908 & 0.814014 & 0.420908 \\
\hline & 50 & 1.886316 & 0.639349 & 0.386316 & 1.742124 & 0.578593 & 0.242124 & 1.800076 & 0.600154 & 0.300076 \\
\hline & 100 & 1.784096 & 0.455022 & 0.284096 & 1.668856 & 0.484527 & 0.168856 & 1.702936 & 0.432923 & 0.202936 \\
\hline & 200 & 1.703712 & 0.356608 & 0.203712 & 1.642288 & 0.351540 & 0.142288 & 1.624206 & 0.318370 & 0.124206 \\
\hline & 400 & 1.626176 & 0.233461 & 0.126176 & 1.619398 & 0.282194 & 0.119398 & 1.571226 & 0.225083 & 0.071226 \\
\hline & 800 & 1.583094 & 0.166228 & 0.083094 & 1.601048 & 0.231125 & 0.101048 & 1.554421 & 0.179775 & 0.054421 \\
\hline \multirow{5}{*}{$\lambda$} & 25 & 0.745644 & 1.620159 & 0.245644 & 0.614386 & 0.916302 & 0.114386 & 0.802302 & 1.925069 & 0.302302 \\
\hline & 50 & 0.789834 & 1.827206 & 0.289834 & 0.688256 & 0.980021 & 0.188256 & 0.724022 & 1.308039 & 0.224022 \\
\hline & 100 & 0.668703 & 1.148297 & 0.168702 & 0.767121 & 1.181886 & 0.267121 & 0.631323 & 0.918628 & 0.131323 \\
\hline & 400 & 0.500787 & 0.547748 & 0.000787 & 0.751948 & 0.888859 & 0.251948 & 0.532055 & 0.562579 & 0.032055 \\
\hline & 800 & 0.445647 & 0.236027 & -0.054353 & 0.677007 & 0.564256 & 0.177007 & 0.503632 & 0.345103 & 0.003632 \\
\hline \multirow{6}{*}{$\gamma$} & 25 & 1.293250 & 1.505832 & 1.243250 & 2.253616 & 1.581109 & 0.753616 & 1.511239 & 1.444601 & 1.011239 \\
\hline & 50 & 1.078543 & 1.444707 & 1.028543 & 1.998239 & 1.049520 & 0.498239 & 1.276449 & 1.236212 & 0.776449 \\
\hline & 100 & 0.809327 & 1.013048 & 0.759326 & 1.857415 & 1.228921 & 0.357415 & 1.031720 & 0.851569 & 0.531720 \\
\hline & 200 & 0.637999 & 0.694046 & 0.587999 & 1.611941 & 0.690544 & 0.111941 & 0.831883 & 0.558033 & 0.331883 \\
\hline & 400 & 0.454666 & 0.482707 & 0.404666 & 1.566871 & 0.491686 & 0.066871 & 0.716428 & 0.401129 & 0.216428 \\
\hline & 800 & 0.364646 & 0.379788 & 0.314646 & 1.534830 & 0.408860 & 0.034830 & 0.671188 & 0.348582 & 0.171188 \\
\hline \multirow{6}{*}{$\theta$} & 25 & 1.803917 & 1.742115 & 0.803917 & 1.749661 & 1.588358 & 0.749661 & 1.742046 & 1.568870 & 0.742046 \\
\hline & 50 & 1.638463 & 1.394404 & 0.638463 & 1.509847 & 1.298293 & 0.509847 & 1.607445 & 1.335610 & 0.607445 \\
\hline & 100 & 1.509126 & 1.134739 & 0.509125 & 1.396531 & 1.100253 & 0.396531 & 1.511317 & 1.127434 & 0.511317 \\
\hline & 400 & 1.446845 & 0.911671 & 0.446845 & 1.188926 & 0.842381 & 0.188926 & 1.391992 & 0.880468 & 0.391992 \\
\hline & 800 & 1.377106 & 0.761436 & 0.377105 & 1.118728 & 0.725644 & 0.118728 & 1.312702 & 0.755088 & 0.312702 \\
\hline & & \multicolumn{3}{|c|}{ IV: $\alpha=1.5, \lambda=1.0, \gamma=1.0, \theta=0.5$} & \multicolumn{3}{|c|}{$\mathrm{V}: \alpha=1.5, \lambda=1.0, \gamma=0.2, \theta=0.5$} & \multicolumn{3}{|c|}{ VI: $\alpha=1.5, \lambda=1.0, \gamma=1.5, \theta=0.5$} \\
\hline \multirow{6}{*}{$\alpha$} & 25 & 1.789584 & 1.170582 & 0.289584 & 1.830471 & 1.073062 & 0.330471 & 1.868657 & 1.452572 & 0.368657 \\
\hline & 50 & 1.629157 & 0.641872 & 0.129157 & 1.728740 & 0.658916 & 0.228740 & 1.715905 & 0.950434 & 0.215905 \\
\hline & 100 & 1.601907 & 0.582973 & 0.101907 & 1.654207 & 0.471052 & 0.154207 & 1.649254 & 0.723506 & 0.149254 \\
\hline & 200 & 1.569387 & 0.390989 & 0.069386 & 1.562855 & 0.362471 & 0.062855 & 1.600683 & 0.399963 & 0.100683 \\
\hline & 400 & 1.563512 & 0.360009 & 0.063512 & 1.545335 & 0.324527 & 0.045335 & 1.580855 & 0.314712 & 0.080855 \\
\hline & 800 & 1.530004 & 0.253489 & 0.030004 & 1.502995 & 0.237065 & 0.002995 & 1.581890 & 0.256117 & 0.081890 \\
\hline \multirow{6}{*}{$\lambda$} & 25 & 1.234825 & 2.566343 & 0.234825 & 1.277434 & 2.653818 & 0.277434 & 1.259803 & 2.161958 & 0.259803 \\
\hline & 50 & 1.206122 & 1.855891 & 0.206122 & 1.553472 & 2.685576 & 0.553472 & 1.244136 & 1.643634 & 0.244135 \\
\hline & 100 & 1.353491 & 2.205158 & 0.353491 & 1.353464 & 2.079207 & 0.353464 & 1.330745 & 1.917436 & 0.330745 \\
\hline & 200 & 1.245384 & 1.411865 & 0.245384 & 1.140746 & 1.876867 & 0.140746 & 1.336354 & 1.411458 & 0.336354 \\
\hline & 400 & 1.294687 & 1.579722 & 0.294687 & 1.160668 & 1.426286 & 0.160668 & 1.205235 & 0.925800 & 0.205235 \\
\hline & 800 & 1.050612 & 0.649950 & 0.050612 & 0.929608 & 0.686878 & -0.070392 & 1.140656 & 0.657567 & 0.140656 \\
\hline \multirow{6}{*}{$\gamma$} & 25 & 2.581599 & 2.439986 & 1.581599 & 2.018081 & 2.528360 & 1.818081 & 3.000350 & 2.870611 & 1.500350 \\
\hline & 50 & 2.279403 & 2.005016 & 1.279403 & 1.808435 & 3.170521 & 1.608435 & 2.688937 & 2.201449 & 1.188937 \\
\hline & 100 & 2.084171 & 1.859060 & 1.084170 & 1.449362 & 1.621622 & 1.249362 & 2.530155 & 2.252898 & 1.030155 \\
\hline & 200 & 1.609610 & 1.012570 & 0.609610 & 1.121505 & 1.151478 & 0.921505 & 2.005328 & 1.052877 & 0.505328 \\
\hline & 400 & 1.494518 & 1.126263 & 0.494518 & 0.893239 & 0.845082 & 0.693239 & 1.857734 & 0.852167 & 0.357733 \\
\hline & 800 & 1.328205 & 0.653356 & 0.328205 & 0.724927 & 0.688594 & 0.524927 & 1.782165 & 0.661829 & 0.282165 \\
\hline \multirow{4}{*}{$\theta$} & 25 & 1.436602 & 1.701088 & 0.936602 & 1.711850 & 2.084528 & 1.211850 & 1.314515 & 1.601855 & 0.814515 \\
\hline & 200 & 0.986895 & 1.048300 & 0.486895 & 1.155493 & 1.183833 & 0.655492 & 0.853204 & 0.924416 & 0.353204 \\
\hline & 400 & 0.885174 & 0.927520 & 0.385174 & 0.960644 & 0.906682 & 0.460644 & 0.806896 & 0.857003 & 0.306896 \\
\hline & 800 & 0.784872 & 0.707049 & 0.284872 & 0.862063 & 0.734907 & 0.362063 & 0.719819 & 0.719558 & 0.219819 \\
\hline
\end{tabular}

\section{Applications}

In this section, we present two real data examples to demonstrate the applicability of the OW-TLLLoGP distribution. We use the maximum likelihood estimation technique to estimate the model parameters. We also make use of the R software for data fitting and model diagnostics. We present the parameters estimates (standard errors in parentheses) and the various goodness of fit statistics in Tables 6 and 7. We considered the following goodness-of-fit statistics to evaluate model performance; -2loglikelihood 
(-2 $\log \mathrm{L})$, Akaike Information Criterion (AIC), Consistent Akaike Information Criterion (AICC), Bayesian Information Criterion (BIC), Cramer von Mises $\left(W^{*}\right)$, Andersen-Darling $\left(A^{*}\right)$, Kolmogorov Simirnov (KS) and its p-value. The smaller the values of the goodness-of-fit statistics, the better the model. Also, the OWTL-LLoGP model was compared to its nested models using the likelihood ratio test. Furthermore, we provide fitted densities and probability plots to demonstrate how the OW-TL-LLoGP model fit the two data sets. The non-nested models considered are the beta odd Lindley-Uniform (BOL-U) distribution and the beta odd Lindley-Exponential (BOL-E) by [12], Kumaraswamy odd Lindley-log logistic (KOL-LLoG) by [9], exponential Lindley odd log-logistic Weibull (ELOLLW) by [23], Topp-Leone-Marshall-Olkin-Weibull (TLMO-W) by [11], Kumaraswamy-Weibull $(\mathrm{KwW})$ by [14], beta-Weibull (BW) by [13], the exponentiated power generalized Weibull (EPGW) distribution [27] and odd exponentiated half logistic-Burr XII (OEHLBXII) [2] distributions. The pdfs of the non-nested models are

$$
f_{\text {TTL }-W}(x ; a, b, \alpha, \lambda)=2 \alpha b a^{b} x^{b-1} e^{-2(a x)^{b}}\left(1-e^{-2(a x)^{b}}\right)^{\alpha-1}\left(1+\lambda-2 \lambda\left(1-e^{-2(a x)^{b}}\right)^{\alpha}\right),
$$

for $a, b, \alpha, \lambda>0$,

$$
\begin{aligned}
f_{\text {ELOLLW }}(x ; \alpha, \beta, \gamma, \theta, \lambda)= & \frac{\alpha \theta^{2} \gamma \lambda^{\gamma} \chi^{\gamma-1} e^{-(\lambda x)^{\gamma}}\left(e^{-(\lambda x)}\right)^{\alpha \theta-1}\left(1-e^{-(\lambda x)^{\gamma}}\right)^{\alpha-1}}{(\theta+\beta)\left(\left(1-e^{-(\lambda x)^{\gamma}}\right)^{\alpha}+e^{-\alpha(\lambda x)^{\gamma}}\right)^{\theta-1}} \\
& \times\left(1-\beta \log \left[\frac{e^{-(\lambda x)^{\gamma}}}{\left(1-e^{-(\lambda x)^{\gamma}}\right)^{\alpha}+e^{-\alpha(\lambda x)^{\gamma}}}\right]\right),
\end{aligned}
$$

for $\alpha, \beta, \gamma, \theta, \lambda>0$,

$$
\begin{aligned}
& f_{\text {KOL-LLoG }}(x ; a, b, \lambda, c)=a b\left[\frac{\lambda^{2}}{(1+\lambda)} \frac{c x^{c-1}}{\left(1+x^{c}\right)^{-1}} \exp \left\{-\lambda \frac{1-\left(1+\chi^{c}\right)^{-1}}{\left(\left(1+x^{c}\right)^{-1}\right)}\right\}\right] \\
& \times\left[1-\frac{\lambda+\left(\left(1+x^{\mathfrak{c}}\right)^{-1}\right)}{(1+\lambda)\left(\left(1+x^{\mathfrak{c}}\right)^{-1}\right)} \exp \left\{-\lambda \frac{\left(1-\left(1+x^{\mathfrak{c}}\right)^{-1}\right)}{\left(\left(1+x^{\mathfrak{c}}\right)^{-1}\right)}\right]^{\mathrm{a}-1}\right. \\
& \times\left(1-\left[1-\frac{\lambda+\left(\left(1+x^{\mathfrak{c}}\right)^{-1}\right)}{(1+\lambda)\left(\left(1+x^{\mathfrak{c}}\right)^{-1}\right)} \exp \left\{-\lambda \frac{\left(1-\left(1+x^{\mathfrak{c}}\right)^{-1}\right)}{\left(\left(1+x^{\mathfrak{c}}\right)^{-1}\right)}\right\}\right]^{\mathrm{a}}\right)^{\mathrm{b}-1},
\end{aligned}
$$

for $a, b, \lambda, c>0$,

$$
f_{B W}(x ; a, b, \alpha, \beta)=\frac{\beta \alpha^{\beta}}{B(a, b)} x^{\beta-1} e^{-b(\alpha x)^{\beta}}\left(1-e^{-(\alpha x)^{\beta}}\right)^{a-1},
$$

for $a, b, \alpha, \beta>0$,

$$
f_{K w W}(x ; a, b, \alpha, \beta)=a b \alpha^{\beta} x^{\beta-1} e^{-(\alpha x)^{\beta}}\left(1-e^{-(\alpha x)^{\beta}}\right)^{a-1}\left(1-\left(1-e^{-(\alpha x)^{\beta}}\right)^{a}\right)^{b-1},
$$

for $a, b, \alpha, \beta>0$,

$$
\begin{aligned}
f_{B O L}-u(x ; a, b, \lambda, \theta)= & \frac{1}{B(a, b)}\left[1-\frac{\lambda+(1-x / \theta)}{(1+\lambda)(1-x / \theta)} \exp \left\{-\lambda \frac{x}{(\theta-x)}\right\}\right]^{a-1} \\
& \times\left[\frac{\lambda+(1-x / \theta)}{(1+\lambda)(1-x / \theta)} \exp \left\{-\lambda \frac{x}{(\theta-x)}\right\}\right]^{b-1} \frac{\lambda^{2}}{(1+\lambda)} \frac{\theta^{2}}{(\theta-x)^{3}} \exp \left\{-\lambda \frac{x}{(\theta-x)}\right\},
\end{aligned}
$$

for $a, b, \lambda>0$ and $0<x<\theta$,

$$
\mathrm{f}_{\text {OEHLBXII }}(x ; \alpha, \lambda, a, b)=\frac{2 \alpha \lambda a b x^{a-1} \exp \left(\lambda\left[1-\left(1+x^{a}\right)^{b}\right]\right)\left(1-\exp \left(\lambda\left[1-\left(1+x^{a}\right)^{b}\right]\right)\right)^{\alpha-1}}{\left(1+\chi^{a}\right)^{-b-1}\left(1+\exp \left(\lambda\left[1-\left(1+\chi^{a}\right)^{b}\right]\right)\right)^{\alpha+1}}
$$

for $\alpha, \lambda, a, b>0$,

$$
\mathrm{f}_{\mathrm{TLMO}-\mathrm{W}}(\mathrm{x} ; \mathrm{b}, \delta, \lambda, \gamma)=\frac{2 \mathrm{~b} \delta^{2} \lambda \gamma \mathrm{x}^{\gamma-1} e^{-2 \lambda x^{\gamma}}}{\left(1-\bar{\delta} e^{-\lambda x^{\gamma}}\right)^{3}}\left[1-\frac{\delta^{2} e^{-2 \lambda x^{\gamma}}}{\left(1-\bar{\delta} e^{-\lambda x^{\gamma}}\right)^{2}}\right]^{\mathrm{b}-1}
$$


for $b, \delta, \lambda, \omega>0$,

$$
f_{E P G W}(x ; \lambda, \gamma, \alpha, \beta)=\frac{\alpha \beta \lambda \gamma \chi^{\gamma-1}\left(1+\lambda x^{\gamma}\right)^{\alpha-1} \exp \left(1-\left(1+\lambda x^{\gamma}\right)^{\alpha}\right)}{\left(1-\exp \left(1-\left(1+\lambda x^{\gamma}\right)^{\alpha}\right)\right)^{(1-\beta)}},
$$

for $\lambda, \gamma, \alpha, \beta>0$ and $x>0$,

$$
\begin{aligned}
f_{B O L-E}(x ; a, b, \lambda, \theta)= & \frac{1}{B(a, b)}\left[1-\frac{\lambda+e^{-\theta x}}{(1+\lambda) e^{-\theta x}} \exp \left\{-\lambda \frac{\left(1-e^{-\theta x}\right)}{e^{-\theta x}}\right\}\right]^{a-1} \\
& \times\left[\frac{\lambda+e^{-\theta x}}{(1+\lambda) e^{-\theta x}} \exp \left\{-\lambda \frac{\left(1-e^{-\theta x}\right)}{e^{-\theta x}}\right\}\right]^{b-1} \frac{\lambda^{2}}{(1+\lambda)} \frac{\left(\theta e^{-\theta x}\right)}{e^{-3 \theta x}} \exp \left\{-\lambda \frac{1-e^{-\theta x}}{e^{-\theta x}}\right\},
\end{aligned}
$$

for $a, b, \lambda, \theta>0$. For the ELOLLOW distribution we considered a case when $\alpha=1$.

\subsection{Chemotherapy treatment data}

The first data set are survival times in years of cancer patients receiving chemotherapy treatment alone. The data set is reported by [1]. The data are $0.047,0.115,0.121,0.132,0.164,0.197,0.203,0.260$, $0.282,0.296,0.334,0.395,0.458,0.466,0.501,0.507,0.529,0.534,0.540,0.641,0.644,0.696,0.841,0.863,1.099$, $1.219,1.271,1.326,1.447,1.485,1.553,1.581,1.589,2.178,2.343,2.416,2.444,2.825,2.830,3.578,3.658,3.743$, $3.978,4.003,4.033$.

The estimated variance-covariance matrix is given by

$$
\left[\begin{array}{cccc}
0.2045 & -0.6991 & 0.0066 & 0.0101 \\
-0.6991 & 2.4133 & -0.0609 & -0.0354 \\
0.0066 & -0.0609 & 0.3729 & 0.0122 \\
0.0101 & -0.0354 & 0.0122 & 0.0142
\end{array}\right]
$$

and the $95 \%$ confidence intervals for the model parameters are given by $\alpha \in[0.3632 \pm 0.8864], \lambda \in[1.4446 \pm$ 3.0448], $\gamma \in[3.2819 \pm 1.1970]$ and $\theta \in\left[9.7836 \times 10^{-7} \pm 0.2337\right]$. Based on the values of the goodness-of-fit statistics $-2 \log \mathrm{L}, \mathrm{AIC}, \mathrm{AICC}, \mathrm{BIC}, \mathrm{W}^{*}, \mathrm{~A}^{*}, \mathrm{KS}$ and its $\mathrm{p}$-value, we conclude that the OW-TL-LLoGP model performs better on chemotherapy treatment survival times data set than the several non-nested models considered in this paper. Also, using the results shown in Table 8, we conclude that the OWTL-LLoGP model performs better than its nested models on chemotherapy treatment survival times data set.

\subsection{Active repair times data}

The second data set represents active repair times (hours) for an airborne communication transceiver. The data was also analyzed by [22]. The data are $0.50,0.60,0.60,0.70,0.70,0.70,0.80,0.80,1.00,1.00,1.00$, $1.00,1.10,1.30,1.50,1.50,1.50,1.50,2.00,2.00,2.20,2.50,2.70,3.00,3.00,3.30,4.00,4.00,4.50,4.70,5.00$, $5.40,5.40,7.00,7.50,8.80,9.00,10.20,22.00,24.50$

The estimated variance-covariance matrix is given by

$$
\left[\begin{array}{cccc}
0.0132 & -0.0387 & -0.2559 & 5.4524 \times 10^{-5} \\
-0.0387 & 0.1248 & 0.7048 & -0.0002 \\
-0.2559 & 0.7048 & 9.0625 & 3.6190 \times 10^{-5} \\
5.4524 \times 10^{-5} & -0.0002 & 3.6190 \times 10^{-5} & 0.0004
\end{array}\right]
$$

and the $95 \%$ confidence intervals for the model parameters are given by $\alpha \in[0.2875 \pm 0.2259], \lambda \in[1.2106 \pm 0.6924], \gamma \in[11.6130 \pm 5.9003]$ and $\theta \in\left[3.1074 \times 10^{-8} \pm 0.0378\right]$. Furthermore, based on the values of the goodness-of-fit statistics $-2 \log$ L, AIC, AICC, BIC, $W^{*}, A^{*}, K S$ and its $p$-value, we conclude that the OW-TL-LLoGP model performs better on repair times data set than the several non-nested models considered in this paper. Also, using the results shown in Table 8, we conclude that the OW-TL-LLoGP model performs better than its nested models on repair times data set. 
Table 6: Parameter estimates and goodness-of-fit statistics for various models fitted for chemotherapy data set.

\begin{tabular}{|c|c|c|c|c|c|c|c|c|c|c|c|c|}
\hline \multirow[b]{2}{*}{ Model } & \multicolumn{4}{|c|}{ Estimates } & \multicolumn{5}{|c|}{ Statistics } & \multirow[b]{2}{*}{$A^{*}$} & \multirow[b]{2}{*}{ KS } & \multirow[b]{2}{*}{$p$-value } \\
\hline & $\alpha$ & $\lambda$ & $\gamma$ & $\theta$ & $-2 \log \mathrm{L}$ & AIC & AICC & BIC & $W^{*}$ & & & \\
\hline OW-TL-LLoGP & $\begin{array}{c}0.3632 \\
(0.4523)\end{array}$ & $\begin{array}{c}1.4446 \\
(1.5535)\end{array}$ & $\begin{array}{c}3.2819 \\
(0.6107)\end{array}$ & $\begin{array}{c}9.7836 \times 10^{-7} \\
(0.1193)\end{array}$ & 114.2 & 122.2 & 123.2 & 129.4 & 0.0438 & 0.3294 & 0.0871 & 0.8550 \\
\hline OW-TL-LLoGP $(\alpha, \gamma, \theta)$ & $\begin{array}{c}0.4606 \\
(0.4606)\end{array}$ & $\begin{array}{l}1 \\
-\end{array}$ & $\begin{array}{c}1.1287 \\
(0.2158)\end{array}$ & $\begin{array}{c}3.6928 \times 10^{-9} \\
(0.0077)\end{array}$ & 149.8 & 155.8 & 156.4 & 161.2 & 0.0867 & 0.5758 & 0.3573 & $1.1970 \times 10^{-5}$ \\
\hline OW-TL-LLoGP $(\alpha, \lambda, \theta)$ & $\begin{array}{c}0.0706 \\
(0.7851)\end{array}$ & $\begin{array}{c}7.5060 \\
(8.2811)\end{array}$ & $\begin{array}{l}1 \\
-\end{array}$ & $\begin{array}{c}3.6584 \times 10^{-8} \\
(0.0211)\end{array}$ & 137.1 & 143.1 & 143.7 & 148.5 & 0.1881 & 1.1638 & 0.2615 & 0.0033 \\
\hline OW-TL-LLoGP $(\lambda, \theta)$ & $\begin{array}{l}1 \\
-\end{array}$ & $\begin{array}{c}0.4458 \\
(0.0429)\end{array}$ & $\begin{array}{l}1 \\
-\end{array}$ & $\begin{array}{c}5.9476 \times 10^{-9} \\
(0.0092)\end{array}$ & 233.6 & 237.6 & 237.9 & 241.2 & 0.0808 & 0.5410 & 0.5973 & $6.6610 \times 10^{-16}$ \\
\hline OW-TL-LLoGP $(\alpha, \theta)$ & $\begin{array}{c}0.4450 \\
(0.0402)\end{array}$ & $\begin{array}{l}1 \\
-\end{array}$ & $\begin{array}{l}1 \\
-\end{array}$ & $\begin{array}{c}2.3754 \times 10^{-8} \\
(0.0173)\end{array}$ & 156.0 & 160.0 & 160.3 & 163.6 & 0.0907 & 0.6006 & 0.3909 & $1.0420 \times 10^{-6}$ \\
\hline OW-TL-LLoGP $(\gamma, \theta)$ & $\begin{array}{l}1 \\
- \\
\end{array}$ & $\begin{array}{l}1 \\
- \\
\end{array}$ & $\begin{array}{c}3.5756 \\
(0.3331)\end{array}$ & $\begin{array}{c}0.7126 \\
(0.5926) \\
\end{array}$ & 155.7 & 159.7 & 160.0 & 163.3 & 0.0662 & 0.4526 & 0.2214 & 0.0204 \\
\hline TTL-W & $\begin{array}{c}\mathrm{a} \\
0.4255 \\
(0.4183)\end{array}$ & $\begin{array}{c}\mathrm{b} \\
0.8883 \\
(0.7586)\end{array}$ & $\begin{array}{c}\alpha \\
1.3940 \\
(2.0105)\end{array}$ & $\begin{array}{c}\lambda \\
0.1166 \\
(0.5849)\end{array}$ & 116.0 & 124.0 & 125.0 & 131.3 & 0.0733 & 0.4953 & 0.1568 & 0.1966 \\
\hline KOL-LLoG & $\begin{array}{c}\mathrm{a} \\
2.4477 \\
(2.8117)\end{array}$ & $\begin{array}{c}\mathrm{b} \\
0.1302 \\
(0.1585)\end{array}$ & $\begin{array}{c}\lambda \\
7.4117 \\
(7.9891)\end{array}$ & $\begin{array}{c}c \\
0.8768 \\
(0.2028)\end{array}$ & 114.9 & 122.9 & 123.9 & 130.2 & 0.0541 & 0.3830 & 0.0969 & 0.7562 \\
\hline BW & $\begin{array}{c}a \\
2.0869 \\
(3.6004)\end{array}$ & $\begin{array}{c}\mathrm{b} \\
11.1746 \\
(32.5967)\end{array}$ & $\begin{array}{c}\lambda \\
0.0683 \\
(0.2789)\end{array}$ & $\begin{array}{c}\mathrm{k} \\
0.6816 \\
(0.6712)\end{array}$ & 116.0 & 124.0 & 125.0 & 131.27 & 0.0662 & 0.4538 & 0.0982 & 0.7411 \\
\hline TLMO-W & $\begin{array}{c}\mathrm{b} \\
1.5663 \\
(1.9293)\end{array}$ & $\begin{array}{c}\delta \\
0.8611 \\
(1.0623)\end{array}$ & $\begin{array}{c}\lambda \\
0.4921 \\
(0.7341)\end{array}$ & $\begin{array}{c}\gamma \\
0.8424 \\
(0.6036)\end{array}$ & 116.1 & 124.1 & 125.1 & 131.3 & 0.0682 & 0.4650 & 0.0991 & 0.7320 \\
\hline BOL-E & $\begin{array}{c}a \\
1.5656 \\
(0.8141)\end{array}$ & $\begin{array}{c}\mathrm{b} \\
0.0617 \\
(0.03425)\end{array}$ & $\begin{array}{c}\lambda \\
118.12 \\
(0.0041)\end{array}$ & $\begin{array}{c}\theta \\
0.0920 \\
(0.0450)\end{array}$ & 115.2 & 123.2 & 124.2 & 130.4 & 1.3332 & 7.4848 & 0.1425 & 0.2917 \\
\hline BOL-U & $\begin{array}{c}0.9574 \\
(0.3187)\end{array}$ & $\begin{array}{c}3.3672 \\
(7.7617)\end{array}$ & $\begin{array}{c}3.8385 \\
(6.8110)\end{array}$ & $\begin{array}{c}17.1583 \\
(30.4900)\end{array}$ & 115.9 & 123.9 & 124.9 & 131.1 & 0.0936 & 0.6198 & 0.1196 & 0.5027 \\
\hline ELOLLW & $\begin{array}{c}\beta \\
0.3337 \\
(1.8652)\end{array}$ & $\begin{array}{c}\lambda \\
0.3243 \\
(0.1816)\end{array}$ & $\begin{array}{c}\theta \\
2.6100 \\
(0.2177)\end{array}$ & $\begin{array}{c}\gamma \\
1.0469 \\
(0.1389)\end{array}$ & 116.2 & 124.2 & 125.2 & 131.5 & 0.0817 & 0.5471 & 0.1098 & 0.6104 \\
\hline $\mathrm{KwW}$ & $\begin{array}{c}a \\
10.5121 \\
(4.9910)\end{array}$ & $\begin{array}{c}b \\
157.1692 \\
(0.0679)\end{array}$ & $\begin{array}{c}\alpha \\
0.5989 \\
(1.36946)\end{array}$ & $\begin{array}{c}\beta \\
0.1637 \\
(0.0464)\end{array}$ & 116.1 & 124.1 & 125.1 & 131.3 & 0.0688 & 0.4686 & 0.1021 & 0.6979 \\
\hline EPGW & $\begin{array}{c}\alpha \\
13.3223 \\
(23.3091)\end{array}$ & $\begin{array}{c}\beta \\
3.8242 \\
(5.3963)\end{array}$ & $\begin{array}{c}\lambda \\
0.0824 \\
(0.1461)\end{array}$ & $\begin{array}{c}\gamma \\
0.3487 \\
(0.2704)\end{array}$ & 115.9 & 123.9 & 124.9 & 131.1 & 0.0689 & 0.4687 & 0.1013 & 0.7073 \\
\hline OEHLBXII & $\begin{array}{c}\alpha \\
0.1230 \\
(0.0443)\end{array}$ & $\begin{array}{c}\lambda \\
0.0190 \\
(0.0402)\end{array}$ & $\begin{array}{c}a \\
6.5252 \\
(2.3417)\end{array}$ & $\begin{array}{c}\mathrm{b} \\
0.5074 \\
(0.1674)\end{array}$ & 117.8 & 125.8 & 126.8 & 133.1 & 0.0862 & 0.5758 & 0.1059 & 0.6552 \\
\hline
\end{tabular}
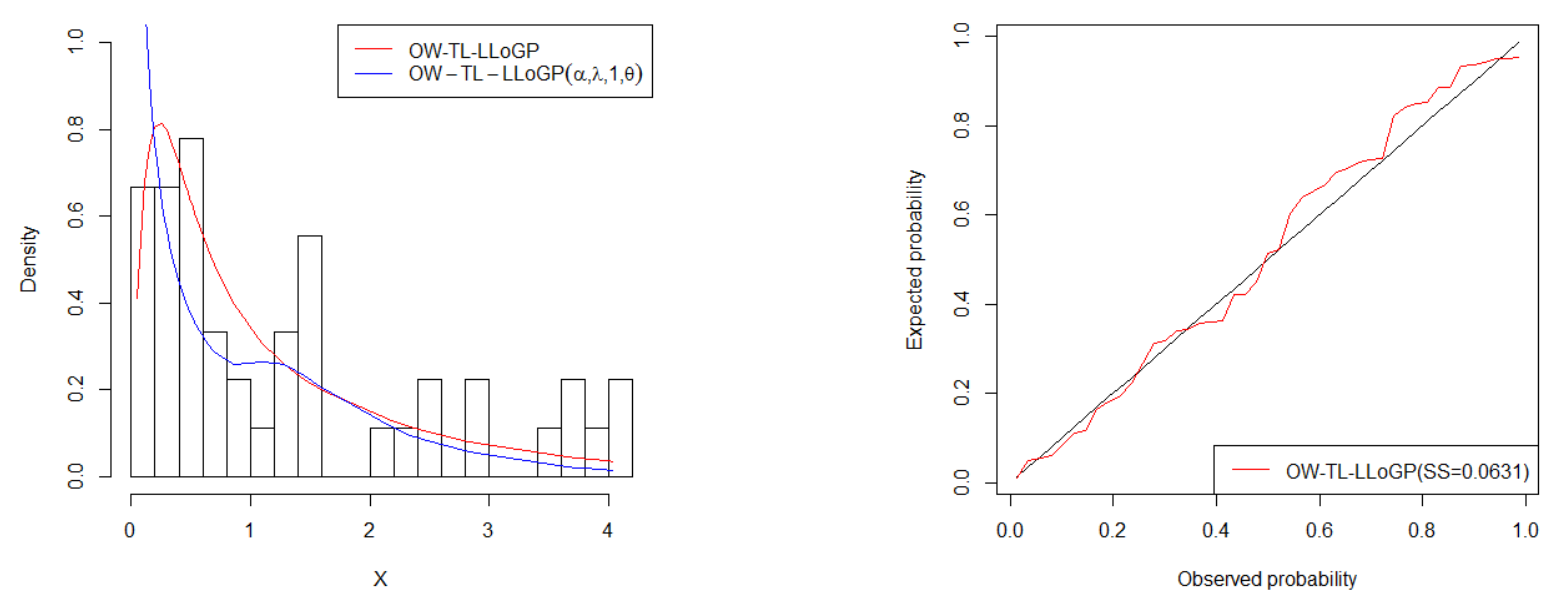

Figure 4: Fitted densities and probability plots for chemotherapy data. 
Table 7: Parameter estimates and goodness-of-fit statistics for various models fitted for active repair times data set.

\begin{tabular}{|c|c|c|c|c|c|c|c|c|c|c|c|c|}
\hline \multirow[b]{2}{*}{ Model } & \multicolumn{4}{|c|}{ Estimates } & \multicolumn{5}{|c|}{ Statistics } & \multirow[b]{2}{*}{$A^{*}$} & \multirow[b]{2}{*}{ KS } & \multirow[b]{2}{*}{$p$-value } \\
\hline & $\alpha$ & $\lambda$ & $\gamma$ & $\theta$ & $-2 \log \mathrm{L}$ & AIC & AICC & BIC & $W^{*}$ & & & \\
\hline OW-TL-LLoGP & $\begin{array}{c}0.2875 \\
(0.1153)\end{array}$ & $\begin{array}{c}1.2106 \\
(0.3533)\end{array}$ & $\begin{array}{l}11.6130 \\
(3.0104)\end{array}$ & $\begin{array}{c}3.1074 \times 10^{-8} \\
(0.00193)\end{array}$ & 197.8 & 205.8 & 206.8 & 213.1 & 0.0267 & 0.1964 & 0.0639 & 0.9918 \\
\hline OW-TL-LLoGP $(\alpha, \gamma, \theta)$ & $\begin{array}{c}0.2703 \\
(0.3171)\end{array}$ & $\begin{array}{l}1 \\
-\end{array}$ & $\begin{array}{c}1.3288 \\
(0.3815)\end{array}$ & $\begin{array}{c}2.3476 \times 10^{-10} \\
(0.0017)\end{array}$ & 266.3 & 272.3 & 272.9 & 277.8 & 0.1279 & 0.8730 & 0.5718 & $1.7140 \times 10^{-13}$ \\
\hline OW-TL-LLoGP $(\alpha, \lambda, \theta)$ & $\begin{array}{c}0.2458 \\
(0.0506)\end{array}$ & $\begin{array}{c}1.1157 \\
(8.2383)\end{array}$ & $\begin{array}{l}1 \\
-\end{array}$ & $\begin{array}{c}3.1762 \times 10^{-9} \\
(0.0081)\end{array}$ & 273.8 & 279.8 & 280.3 & 285.3 & 0.1486 & 1.0047 & 0.6002 & $8.1050 \times 10^{-15}$ \\
\hline OW-TL-LLoGP $(\lambda, \theta)$ & $\begin{array}{l}1 \\
-\end{array}$ & $\begin{array}{c}0.2142 \\
(0.0226)\end{array}$ & $\begin{array}{l}1 \\
-\end{array}$ & $\begin{array}{c}2.0769 \times 10^{-8} \\
(0.0156)\end{array}$ & 409.2 & 413.2 & 413.5 & 416.9 & 0.0829 & 0.5633 & 0.8717 & $<2.2000 \times 10^{-16}$ \\
\hline OW-TL-LLoGP $(\alpha, \theta)$ & $\begin{array}{c}0.2616 \\
(0.0237)\end{array}$ & $\begin{array}{l}1 \\
-\end{array}$ & $\begin{array}{l}1 \\
-\end{array}$ & $\begin{array}{c}1.2944 \times 10^{-8} \\
(0.0125)\end{array}$ & 276.2 & 280.2 & 280.5 & 283.8 & 0.1382 & 0.9388 & 0.6101 & $2.6650 \times 10^{-15}$ \\
\hline OW-TL-LLoGP $(\gamma, \theta)$ & $\begin{array}{l}1 \\
- \\
\end{array}$ & $\begin{array}{l}1 \\
- \\
\end{array}$ & $\begin{array}{l}14.7853 \\
(0.9128)\end{array}$ & $\begin{array}{c}1.8048 \\
(0.5746)\end{array}$ & 442.7 & 446.7 & 447.0 & 450.4 & 0.1028 & 0.8551 & 0.3435 & $3.855 \times 10^{-5}$ \\
\hline TTL-W & $\begin{array}{c}\mathrm{a} \\
0.4255 \\
(0.4183) \\
\end{array}$ & $\begin{array}{c}\mathrm{b} \\
0.8883 \\
(0.7586) \\
\end{array}$ & $\begin{array}{c}\alpha \\
1.3940 \\
(2.0105) \\
\end{array}$ & $\begin{array}{c}\lambda \\
0.1166 \\
(0.5849) \\
\end{array}$ & 116.0 & 124.0 & 125.0 & 131.3 & 0.0733 & 0.4953 & 0.1568 & 0.1966 \\
\hline TTL-W & $\begin{array}{c}\mathrm{a} \\
97.1294 \\
(22.2807)\end{array}$ & $\begin{array}{c}\mathrm{b} \\
0.1940 \\
(0.0186)\end{array}$ & $\begin{array}{c}\alpha \\
159.0913 \\
(26.1871)\end{array}$ & $\begin{array}{c}\lambda \\
0.0000 \\
(0.6043)\end{array}$ & 199.2 & 207.2 & 208.2 & 214.6 & 0.0650 & 0.4295 & 0.9714 & $<2.2 \times 10^{-16}$ \\
\hline KOL-LLoG & $\begin{array}{c}\mathrm{a} \\
10.0147 \\
(7.8294) \\
\end{array}$ & $\begin{array}{c}\mathrm{b} \\
0.2197 \\
(0.2527) \\
\end{array}$ & $\begin{array}{c}\lambda \\
4.2849 \\
(2.3212) \\
\end{array}$ & $\begin{array}{c}c \\
0.5625 \\
(0.1963) \\
\end{array}$ & 199.1 & 207.1 & 208.1 & 214.4 & 0.0409 & 0.2792 & 0.0890 & 0.8590 \\
\hline BW & $\begin{array}{c}a \\
284.5 \\
(2.5324)\end{array}$ & $\begin{array}{c}\mathrm{b} \\
84.2640 \\
(6.1781)\end{array}$ & $\begin{array}{c}\lambda \\
392.94 \\
(0.1610)\end{array}$ & $\begin{array}{c}\mathrm{k} \\
0.0588 \\
(0.0063)\end{array}$ & 200.2 & 208.2 & 209.2 & 215.5 & 0.0537 & 0.3426 & 0.0975 & 0.7747 \\
\hline TLMO-W & $\begin{array}{c}\mathrm{b} \\
11.2910 \\
(0.0016)\end{array}$ & $\begin{array}{c}\delta \\
0.0988 \\
(0.1983)\end{array}$ & $\begin{array}{c}\lambda \\
0.6171 \\
(0.9128)\end{array}$ & $\begin{array}{c}\gamma \\
0.3938 \\
(0.1880)\end{array}$ & 198.9 & 206.9 & 207.9 & 214.2 & 0.0412 & 0.2571 & 0.0753 & 0.9569 \\
\hline BOL-E & $\begin{array}{c}\mathrm{a} \\
0.9318 \\
\left(1.1817 \times 10^{-9}\right)\end{array}$ & $\begin{array}{c}\mathrm{b} \\
42.5400 \\
\left(2.6162 \times 10^{-11}\right)\end{array}$ & $\begin{array}{c}\lambda \\
72.0250 \\
\left(1.5651 \times 10^{-11}\right)\end{array}$ & $\begin{array}{c}\theta \\
8.5418 \times 10^{-5} \\
\left(1.3041 \times 10^{-5}\right)\end{array}$ & 209.9 & 217.9 & 218.8 & 225.2 & 0.1434 & 0.9949 & 0.1458 & 0.2826 \\
\hline BOL-U & $\begin{array}{c}0.9511 \\
(0.1797)\end{array}$ & $\begin{array}{c}1.2493 \\
(0.2487)\end{array}$ & $\begin{array}{c}1.5868 \times 10^{5} \\
\left(1.8674 \times 10^{-6}\right)\end{array}$ & $\begin{array}{c}7.4059 \times 10^{5} \\
\left(4.0012 \times 10^{-7}\right)\end{array}$ & 209.9 & 217.9 & 218.9 & 225.3 & 0.1441 & 0.9997 & 0.1499 & 0.2519 \\
\hline ELOLLW & $\begin{array}{c}\beta \\
1.9748 \times 10^{-5} \\
(1.5701)\end{array}$ & $\begin{array}{c}\lambda \\
0.0576 \\
(0.0259)\end{array}$ & $\begin{array}{c}\theta \\
4.3375 \\
\left(4.2745 \times 10^{-4}\right)\end{array}$ & $\begin{array}{c}\gamma \\
0.8986 \\
(0.0958)\end{array}$ & 208.9 & 216.9 & 217.9 & 224.3 & 0.1298 & 0.9009 & 0.1204 & 0.5170 \\
\hline KwW & $\begin{array}{c}\mathrm{a} \\
11.1209 \\
(10.4798)\end{array}$ & $\begin{array}{c}\mathrm{b} \\
0.2329 \\
(0.3015)\end{array}$ & $\begin{array}{c}\alpha \\
9.5744 \\
(8.8854)\end{array}$ & $\begin{array}{c}\beta \\
0.5748 \\
(0.2021)\end{array}$ & 198.9 & 206.9 & 207.9 & 214.3 & 0.0389 & 0.2637 & 0.0942 & 0.8093 \\
\hline EPGW & $\begin{array}{c}\alpha \\
0.5175 \\
(0.0091)\end{array}$ & $\begin{array}{c}\beta \\
77.6080 \\
\left(5.7573 \times 10^{-5}\right)\end{array}$ & $\begin{array}{c}\lambda \\
22.7070 \\
\left(2.0097 \times 10^{-4}\right)\end{array}$ & $\begin{array}{c}\gamma \\
0.3682 \\
(0.0403)\end{array}$ & 199.1 & 207.2 & 208.2 & 214.6 & 0.0449 & 0.2751 & 0.0795 & 0.9331 \\
\hline OEHLBXII & $\begin{array}{c}\alpha \\
0.1711 \\
(0.0665)\end{array}$ & $\begin{array}{c}\lambda \\
0.0498 \\
(0.0955)\end{array}$ & $\begin{array}{c}\mathrm{a} \\
11.0701 \\
(5.2751)\end{array}$ & $\begin{array}{c}\mathrm{b} \\
0.1235 \\
(0.0434)\end{array}$ & 206.5 & 214.5 & 215.4 & 221.8 & 0.0551 & 0.3817 & 0.0922 & 0.8286 \\
\hline
\end{tabular}
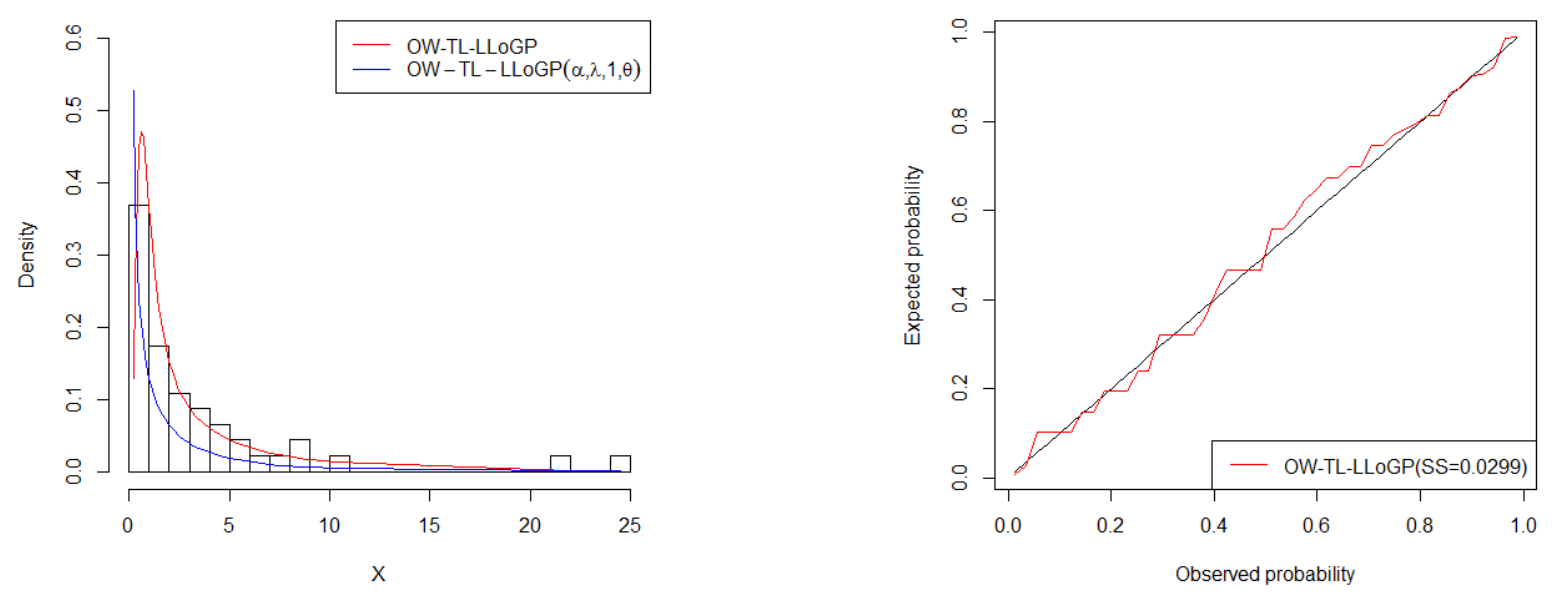

Figure 5: Fitted densities and probability plots for repair times data.

\subsubsection{Likelihood ratio test results}

Table 8 shows the results of the likelihood ratio test. 
Table 8: Likelihood ratio test results for active repair times data.

\begin{tabular}{cccc}
\hline & & Chemotherapy Data Set & Repair Times Data Set \\
\hline Model & $\mathrm{df}$ & $\chi 2(\mathrm{p}$-value $)$ & $\chi 2(\mathrm{p}$-value $)$ \\
\hline OW-TL-LLoGP $(\alpha, \gamma, \theta)$ & 1 & $35.6(<0.00001)$ & $68.5(<0.00001)$ \\
OW-TL-LLoGP $(\alpha, \lambda, \theta)$ & 1 & $22.9(<0.00001)$ & $76.0(<0.00001)$ \\
OW-TL-LLoGP $(\lambda, \theta)$ & 2 & $119.4(<0.00001)$ & $211.4(<0.00001)$ \\
OW-TL-LLoGP $(\alpha, \theta)$ & 2 & $41.8(<0.00001)$ & $78.4(<0.00001)$ \\
OW-TL-LLoGP $(\gamma, \theta)$ & 2 & $41.5(<0.00001)$ & $244.9(<0.00001)$ \\
\hline
\end{tabular}

We conclude from the likelihood ratio test that the OW-TL-LLoGP model performs better than its nested models on chemotherapy treatment survival times and repair times data sets.

\section{Conclusions}

A new generalization of the odd Weibull-Topp-Leone-G family of distributions called the odd WeibullTopp-Leone-G power series (OW-TL-GPS) family of distributions is developed. Statistical properties of the new distribution were also derived. We also derive the maximum likelihood estimates of the proposed model. Some special cases for the new family of distributions were also considered. We conducted a simulation study to evaluate consistency of the maximum likelihood estimates. Two real data examples were also considered to demonstrate usefulness of the new proposed family of distributions. The new distribution performs better than several non-nested models.

\section{Acknowledgment}

We are grateful to the editor and referees for the improved version of this paper.

\section{References}

[1] O. O. Aalen, Heterogeneity in Survival Analysis, Statistics in Medicine, 7 (1988), 1121-1137. 6.1

[2] M. A. D. Aldahlan, A. Z. Afify, The odd exponentiated half-logistic Burr XII distribution, Pak. J. Stat. Oper. Res., 14 (2018), 305-317. 1,6

[3] M. Alizadeh, A. Z. Afify, M. S. Eliwa, S. Ali, The Odd Log-Logistic Lindley-G Family of Distributions: Properties, Bayesian and Non-Bayesian Estimation with Applications, Comput. Stat., 35 (2020), 281-308. 1

[4] Z. A. Al-Saiary, R. A. Bakoban, The Topp-Leone Generalized Inverted Exponential Distribution with Real Data Applications, Entropy, 22 (2020), 15 pages. 1

[5] A. Al-Shomrani, O. Arif, A. Shawky, S. Hanif, M. Q. Shahbaz, Topp-Leone family of distributions: Some properties and application, Pak. J. Stat. Oper. Res., 12 (2016), 443-451. 1, 1

[6] R. G. Aryal1, E. M. Ortega, G. G. Hamedani, H. M. Yousof, The Topp-Leone Generated Weibull Distribution: Regression Model, Characterizations and Applications, Int. J. Stat. Prob., 6 (2017), 126-141. 1

[7] H. Bidram, V. Nekoukhou, Double Bounded Kumaraswamy-Power Series Class of Distributions, SORT, 37 (2013), 211230. 1

[8] F. Chipepa, B. Oluyede, S. Chamunorwa, The odd Weibull-Topp-Leone-G Family of Distributions: Model, Properties and Applications, To Appear, (2020). 1

[9] F. Chipepa, B. O. Oluyede, B. Makubate, A New Generalized Family of Odd Lindley-G Distributions with Application, Int. J. Stat. Prob., 8 (2019), 1-23. 6

[10] F. Chipepa, B. Oluyede, B. Makubate, The Odd Generalized Half-Logistic Weibull-G Family of Distributions: Properties and Applications, J. Stat. Model. Theor. Appl., 2020 (2020), 25 Pages. 1

[11] F. Chipepa, B.Oluyede, B. Makubate, The Topp-Leone-Marshall-Olkin-G Family of Distributions with Applications, Int. J. Stat. Prob., 9 (2020), 15-32. 1, 6

[12] F. Chipepa, B. Oluyede, B. Makubate, A. Fagbamigbe, The Beta Odd Lindley-G Family of Distributions with Applications, J. Prob. Stat. Sci., 17 (2019), 51-84. 6

[13] G. M. Cordeiro, A. E. Gomes, C. Q. da-Silva, E. M. M. Ortega, The Beta Exponentiated Weibull Distribution, J. Stat. Comput. Simul., 83 (2013), 114-138. 6 
[14] G. M. Cordeiro, E. M. M. Ortega, S. Nadarajaah, The Kumaraswamy Weibull Distribution with Application to Failure Data, J. Franklin Inst., 347 (2010), 1399-1429. 6

[15] G. M. Cordeiro, R. B. Silva, The Complementary Extended Weibull Power Series Class of Distributions, Ciência e Natura, 36 (2014), 1-13. 1

[16] F. Gomes-Silva, A. Percontini, E. de Brito, M. W. Ramos, R Venáncio, G. M. Cordeiro, The odd Lindley-G family of distributions, Aust. J. Stat., 46 (2017), 65-87. 1

[17] I. S. Gradshetyn, I. M. Ryzhik, Tables of Integrals, Series and Products, Sixth edition, Academic Press, San Diego, (2000). 2.6, 2.7

[18] M. R. Gurvich, A. T. DiBenedetto, S. V. Ranade, A New Statistical Distribution for Characterizing the Random Strength of Brittle Materials, J. Mat. Sci., 32 (1997), 2559-2564. 1

[19] S. S. Harandi, M. H. Alamatsaz, Generalized Linear Failure Rate Power Series Distribution, Comm. Statist. Theory Methods, 45 (2016), 2204-2227. 1

[20] M. Ibrahim, H. Yousof, Transmuted Topp-Leone Weibull Lifetime Distribution: Statistical Properties and Different Method of Estimation, Pak. J. Stat. Oper. Res., 16 (2020), 501-515. 1

[21] N. L. Johnson, S. Kotz, N. Balakrishnan, Continuous univariate distributions, John Wiley \& Sons, New York, (1994). 1

[22] B. Jorgensen, Statistical Properties of the Generalized Inverse Gaussian Distribution, Springer-Verlag, New York, (1982). 6.2

[23] M. C. Korkmaz, H. M. Yousof, G. G. Hamedani, The Exponential Lindley Odd Log-Logistic-G Family: Properties, Characterizations and Applications, J. Stat. Theory Appl., 17 (2018), 554-571. 6

[24] E. Mahmoudi, A. A. Jafari, Generalized Exponential-Power Series Distributions, Comput. Statist. Data Anal., 56 (2012), 4047-4066. 1

[25] E. Mahmoudi, A. A. Jafari, The Compound Class of Linear Failure Rate-Power Series Distributions: Model, Properties, and Applications, Comm. Statist. Simulation Comput., 46 (2017), 1414-1440. 1

[26] A. L. Morais, W. Barreto-Souza, A Compound Class of Weibull and Power Series Distributions, Comput. Statist. Data Anal., 55 (2011), 1410-1425. 1

[27] F. A. Pena-Ramirez, R. R. Guerra, G. M. Cordeiro, P. R. D. Marinho, The Exponentiated Power Generalized Weibull: Properties and Applications, An. Acad. Brasil. Cienc., 90 (2018), 2553-2577. 6

[28] A. Rényi, On Measures of Entropy and Information, Proc. 4th Berkeley Sympos. Math. Statist. and Prob., Univ. California Press, Berkeley, 1961 (1961), 547-561. 2.7

[29] C. E. Shannon, Prediction and Entropy of Printed English, The Bell System Technical J., 30 (1951), 50-64. 2.7

[30] R. B. Silva, M. Bourguignon, C. R. B. Dias, G. M. Cordeiro, The Compound Class of Extended Weibull Power Series Distributions, Comput. Statist. Data Anal., 58 (2013), 352-367. 1

[31] R. B. Silva, G. M. Cordeiro, The Burr XII Power Series Distributions: A New Compounding Family, Braz. J. Probab. Stat., 29 (2015), 565-589. 1

\section{Appendix}

https://drive.google.com/file/d/1swmGfP4uBhnzIAVMMmTQEaXHx94zJkeB/view?usp=sharing 